\title{
Transmission electron microscopy study of very low-grade metamorphic evolution in Neoproterozoic pelites of the Puncoviscana formation (Cordillera Oriental, NW Argentina)
}

\author{
M. DO CAMPO ${ }^{1, *}$ AND F. NIETO ${ }^{2}$ \\ ${ }^{1}$ Instituto de Geocronología y Geología Isotópica and Facultad de Ciencias Exactas y Nautrales, U.B.A., Pabellón \\ INGEIS, Ciudad Universitaria, 1428-Buenos Aires, Argentina, and ${ }^{2}$ Departamento de Mineralogía y Petrología and \\ I.A.C.T., Universidad de Granada-CSIC, Avda. Fuentenueva s/n, 18002-Granada, Spain
}

(Received 17 April 2003; revised 19 May 2003)

\begin{abstract}
The Puncoviscana Formation, largely cropping out in NW Argentina, is mainly composed of a pelite-greywacke turbidite sequence affected by incipient regional metamorphism and polyphase deformation. Metapelites, composed mainly of quartz, albite, dioctahedral mica and chlorite, were sampled in the Lules-Puncoviscana and Choromoro belts. Lattice-fringe images, selected area electron diffraction and analytical electron microscopy analyses, coupled with previous data from white mica crystallinity index, indicate a state of reaction progress for Puncoviscana slates consistent with medium anchizone- to epizone-grade metamorphism. The $2 M$ polytype prevails in dioctahedral micas, coexisting in a few cases with the $1 M d$ polytype as a consequence of lack of equilibrium. The $2 M$ polytype coexists with $3 T$ in two slates and long-range four-layer and ten-layer stacking sequences were identified in another sample. Samples with $3 T$ and long-range stacking sequences present $\mathbf{b}$ values characteristic of intermediate-high pressure metamorphism and ordered chlorites $(1 L, 2 L, 3 L$ and $7 L)$ prevail.

Based on the $\mathrm{Si}$ contents of dioctahedral micas and considering peak temperatures of $\sim 350-400^{\circ} \mathrm{C}$, pressures from $5 \mathrm{kbar}$ and $5-7 \mathrm{kbar}$ were derived for metapelites from the LulesPuncoviscana and Choromoro belts, respectively. These values agree with facies series derived from the $\mathbf{b}$ values. Micas with a wide range of phengitic substitution, as evidenced by $\mathrm{Fe}+\mathrm{Mg}$ and $\mathrm{Si}$ contents, coexist. These variations could not arise from the disturbing effect of detrital white K-mica because TEM evidence indicates that they are absent or represent $<10 \%$ of the mica population. Thus, compositional variations suggest that dioctahedral micas of individual slates crystallized at different pressure conditions in response to the $P-T$ path of the metamorphism. Moreover, in several biotite-free slates the illite crystallinity (IC) values lead to an underestimation of the metamorphic grade attained in these rocks.

The coexistence of IC corresponding to anchizone and the occurrence of biotite in some slates and felsic metavolcanic rocks intercalated in the Puncoviscana metasediments are interpreted to be the result of a metamorphic path including a relatively high-pressure/low-temperature (HP/LT) event, followed by a lower-pressure overprint possibly at higher temperatures than the HP/LT event. Small micas formed during the high-pressure stage would prevail in the $<2 \mu \mathrm{m}$ fraction, producing anchizone IC.
\end{abstract}

Keywords: Central Andes, Puncoviscana Formation, intermediate Na-K dioctahedral micas, wonesite, mixed layers, illite crystallinity.

* E-mail: marga@ingeis.uba.ar

DOI: $10.1180 / 0009855033840109$ 
The reconstruction of $P-T$ paths for medium- to high-grade rocks is a common objective in petrological studies. However, in clastic sequences affected by very low- to low-grade metamorphism these variables and particularly their temporal evolution are difficult to establish, often because of a lack of reliable indicator minerals. Moreover, considerable controversy exists about whether equilibrium conditions are reached in incipient metamorphism. Merriman \& Peacor (1999) compiled considerable evidence of chemical and textural disequilibrium in pelites and metapelites formed at sub-greenschist conditions that invalidate the use of clay-mineral-related 'geothermometers' for accurate determination of temperature. However, the same authors recognized that mineral transitions in pelites generally follow well-constrained sequences as a function of metamorphic grade. They pointed out that there is a general trend to decreasing disorder with increasing grade, which is consistent with a series of prograde transitions in which pelites pass through a sequence of metastable states as they approach stable chemical equilibrium. In this context, Merriman \& Peacor (1999) postulated that the specific state of a given system can be characterized as corresponding to a "state of reaction progress" defined by data obtained by X-ray diffraction (XRD) or transmission electron microscopy (TEM).

Fine-grained rocks, in which intergrown phyllosilicates are the dominant constituents, require sophisticated techniques for study, such as highresolution transmission electron microscopy (HRTEM) and analytical electron microscopy (AEM).

The purpose of this paper is to establish the $P-T$ conditions for metapelitic rocks of the Neoproterozoic Puncoviscana Formation, affected by incipient regional metamorphism, based on the detailed study of representative samples at TEM scale coupled with previously published data on the crystallinity index and b parameter of white mica (Do Campo et al., 1998; Do Campo, 1999a,b).

The Puncoviscana Formation crops out extensively in the Cordillera Oriental and to a lesser extent on the eastern border of Puna (Salta and Jujuy Provinces), in NW Argentina. The comprehension of its metamorphic evolution is an important key for the understanding of the Neoproterozoic-Cambric evolution of South America. In addition, a better knowledge of its metamorphic evolution outlined from mineralogical data would provide a constraint for further geodynamic models of the area.

The HRTEM and AEM techniques have been employed in recent years to study numerous sequences affected by incipient metamorphism (Lee et al., 1984; Lee \& Peacor, 1985; Lee et al., 1986; Dalla Torre et al., 1996; Dong \& Peacor, 1996; López Munguira \& Nieto, 2000; Warr \& Nieto, 1998; Schmidt and Livi, 1999). A comprehensive review can be found in Merriman \& Peacor (1999).

\section{GEOLOGY AND SAMPLE DESCRIPTIONS}

The Puncoviscana Formation (Turner, 1960), which constitutes the basement of the study area, is mainly composed of a pelite-greywacke turbidite sequence, with subordinate sandstone and locally interbedded conglomerates, shelf limestone and volcanic rocks (Omarini, 1983; Omarini \& Baldis, 1984; Jezek, 1990). This unit was affected by polyphase deformation and has eastern and western tectonic units, tending NS which show contrasting tectonic evolution (Mon \& Hongn, 1991, 1996) (Fig. 1). The eastern Lules-Puncoviscana belt is characterized by asymmetric folds overturned to the west, with concomitant development of an axial plane cleavage. A second superposed deformation produced a crenulation cleavage and associated microfolding, which affects the bedding and the axial plane cleavage. The western Choromoro belt is marked by tight chevron folds overturned to the east, accompanied by an east-dipping axial plane foliation. Superimposed on these folds are other cleavage surfaces that are best developed in the western part of the Choromoro belt. The main deformation of the Puncoviscana Formation occurred during the latest Proterozoic-earliest Cambrian Braziliano orogeny (Ramos \& Basei, 1997; Do Campo, 1999b).

The Santa Rosa de Tastil Granitic Complex is an epizonal post-tectonic body that developed a narrow contact zone that comprises an inner hornfels aureole and an outer zone of semi-metamorphosed pelites (Kilmurray et al., 1974). However, only part of this complex batholith belongs to the basement, because recent investigations have demonstrated that the red granite facies intrude Eopalaeozoic sediments (Hongn et al., 2001b). The Puncoviscana Formation is uncomformably overlain by Cambrian sandstone of the Meson Group. 


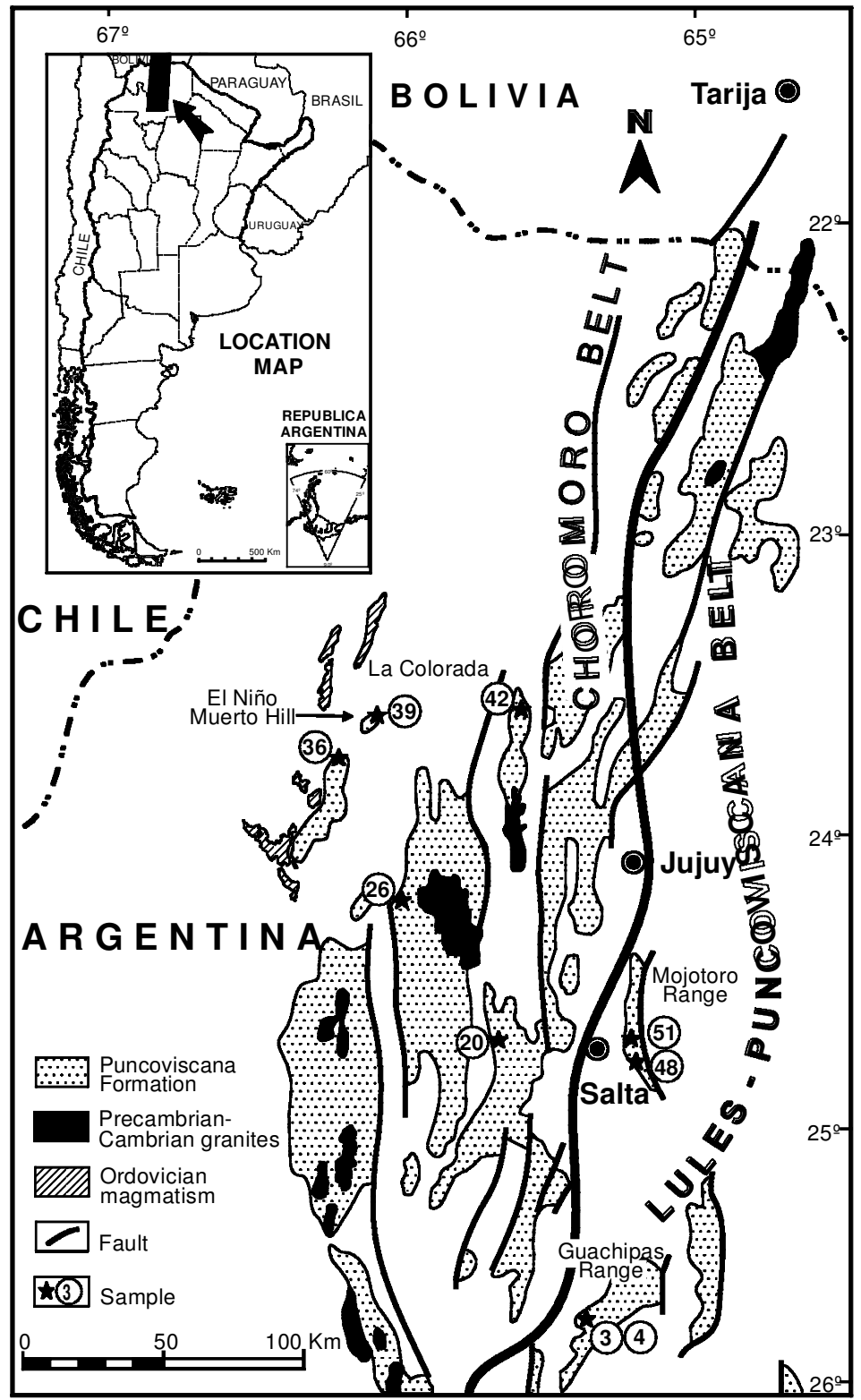

FIG. 1. Geological sketch and location map.

Illite crystallinity values for Lules Puncoviscana belt and the Puna sector of Choromoro belt are in the range of high anchizone and the anchizoneepizone boundary (Table 1, data from Do Campo et al., 1998; Do Campo, 1999a) whereas values for the Cordillera Oriental section of Choromoro belt correspond to anchizone showing a slightly higher average value and more scattering (range 0.36 to $0.25^{\circ} \Delta 2 \theta$ ), (Table 1). The medium anchizonal to epizonal grade indicated by IC data is in agreement with previous results (Toselli \& Rossi de Toselli, 1982; Toselli, 1990).

The presence of prograde biotite in several metapelites from the Sierra del Cobre area and felsic metavolcanic rocks intercalated in the sedimentary sequence at El Niño Muerto Hill 
indicates that greenschist-facies conditions were reached in the eastern border of Puna. However, according to Hongn et al. (2001a), Ordovician magmatic bodies produced a strong metamorphic overprint on volcano-sedimentary rocks in $\mathrm{La}$ Colorada area $15-25 \mathrm{~km}$ north of Cerrillos School; therefore the presence of biotite could also be interpreted as the result of Ordovician overprint.

The nine studied metapelites are mainly composed of detrital quartz and albite plus newly formed phyllosilicates (Table 1). Clasts were reduced in size by pressure solution, alteration and breakdown, which produced elongated quartz and plagioclase grains with preferred orientation. Newly formed white mica and chlorite flakes, $10-50 \mu \mathrm{m}$ in length, show preferred alignment that give place to a continuous slate cleavage in phyllosilicate-rich metapelites (PU4, PU26, PU36 and PU39) and a discontinuous schistosity in quartz-plagioclase rich samples (PU3, PU20, PU42 and PU51). Metamorphic biotite lying sub-parallel to the main foliation was optically identified in several slates from the eastern border of Puna and then corroborated by quantitative analysis at SEM and TEM scale (Do Campo, 1999b). Interleaved phyllosilicate grains composed of chlorite and muscovite or chlorite and biotite can be optically recognized in many rocks. As accessory minerals, zircon, apatite, ilmenite, hematite, iron-titanium oxides and monazite are present. Figure 2 presents BSE images which illustrate the typical texture of an anchizonal and a biotite-bearing Puncoviscana metapelite.

Samples PU3 and PU4 are representative of slates from the Puncoviscana Formation in the Guachipas Range, while PU48 and PU51 correspond to the northernmost Mojotoro Range. Metalimolite PU51 shows curved crystals of trioctahedral mica up to $350 \mu \mathrm{m}$ long and interleaved phyllosilicate grains composed of chlorite + trioctahedral mica as well as chlorite + phengite (Do Campo, 1999b). Samples PU20, PU26 and PU42 correspond to the Choromoro belt in the Cordillera Oriental area; the last one was chosen because it presents a markedly higher IC value (see Table 1), it was also the only metapelite in which minor amounts of detrital K-feldspar were identified at SEM scale. In turn, samples PU36 and PU39 are representative of greenschist-facies slates from the eastern border of Puna. The occurrence of prograde biotite in several slates from this area, including slate PU36 (Cerrillos School), was corroborated by quantitative analysis at SEM scale (Do Campo, 1999b).

\section{TRANSMISSION ELECTRON MICROSCOPY}

Representative samples for TEM observations were chosen based on prior observations by optical microscopy and scanning electron microscopy (SEM). Samples were prepared as Canada Balsam-mounted thin sections oriented approximately normal to bedding and slaty cleavage. They were further thinned using a Gatan 600 ion mill and carbon-coated for TEM observation with a Philips CM-20 scanning transmission electron microscope (STEM) equipped with an ultra-thin window EDX detector (Centro de Instrumentación Científica, C.I.C., Granada University).

Quantitative analyses (AEM) were obtained only from thin edges, using a $70 \AA$ beam diameter and a $1000 \times 200 \AA$ scanning area, with the long axis oriented parallel to the phyllosilicate packets. The

Table 1. Mineral data for Puncoviscana Formation metapelites. Illite crystallinity index from Do Campo (1999a), expressed in CIS scale (Warr \& Rice, 1994). Limiting values for the low-grade and high-grade boundary of the anchizone are $0.42^{\circ}$ and $0.25^{\circ} \Delta 2 \theta$ respectively. $(n)=$ number of samples. Mineral associations do not necessarily represent equilibrium assemblages. Mineral abbreviations after Kretz (1983).

\begin{tabular}{|c|c|c|c|c|}
\hline \multirow{2}{*}{$\begin{array}{l}\text { Tectonic } \\
\text { unit }\end{array}$} & \multirow{2}{*}{$\begin{array}{l}\text { Geological } \\
\text { zone }\end{array}$} & \multirow[t]{2}{*}{ Mineralogy } & \multicolumn{2}{|c|}{$\mathrm{IC}\left({ }^{\circ} \Delta 2 \theta\right)$} \\
\hline & & & Range & Average $(n)$ \\
\hline $\begin{array}{l}\text { Lules- } \\
\text { Puncoviscana belt }\end{array}$ & $\begin{array}{l}\text { Cordillera } \\
\text { Oriental }\end{array}$ & Phen $+\mathrm{Chl} \pm \mathrm{Sm}$ & $0.31-0.24^{\circ}$ & $0.28^{\circ} \pm 0.02$ \\
\hline Choromoro belt & $\begin{array}{l}\text { Cordillera } \\
\text { Oriental }\end{array}$ & Phen + Chl & $0.36-0.25^{\circ}$ & $0.30^{\circ} \pm 0.03$ \\
\hline & Puna & $\mathrm{Phen}+\mathrm{Chl} \pm \mathrm{Bt} \pm \mathrm{Sm}$ & $0.30-0.24^{\circ}$ & $0.27^{\circ} \pm 0.02$ \\
\hline
\end{tabular}



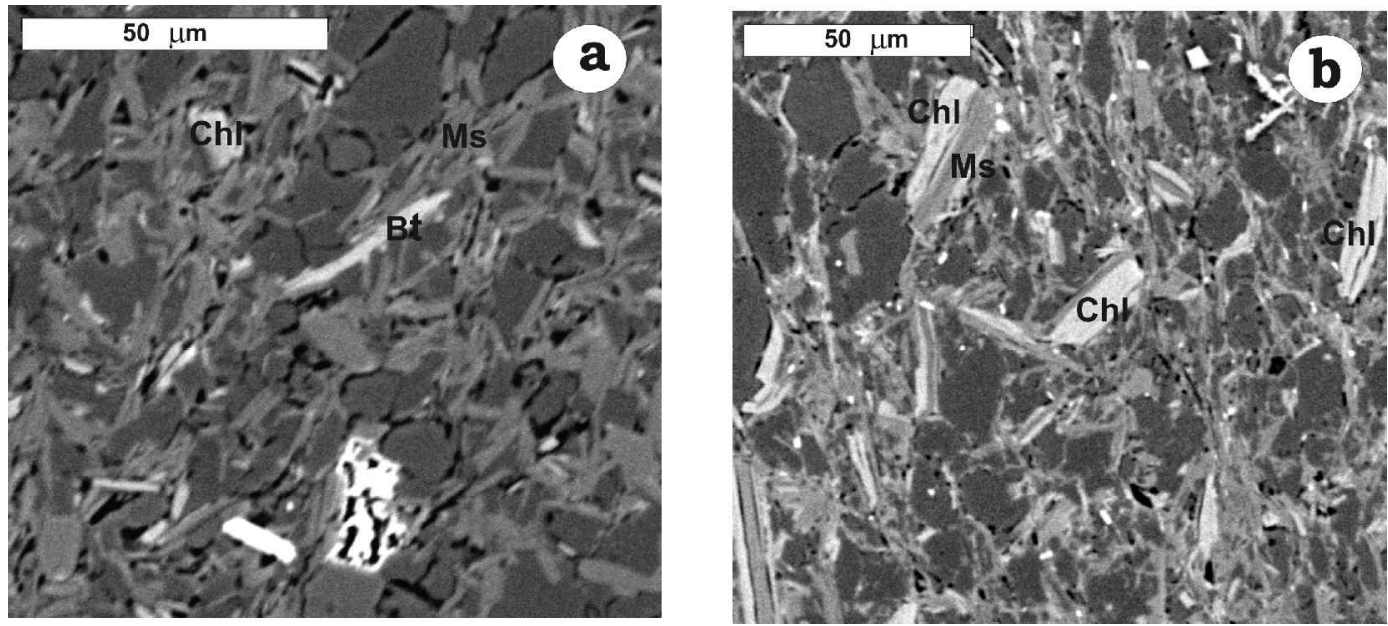

FIG. 2. BSE images of Puncoviscana metapelites: (a) biotite flake parallel to slate cleavage in an epizonal slate, (b) interleaved phyllosilicate grains of chlorite + muscovite in an anchizonal slate. Grains of intermediate contrast are quartz and albite.

sample was tilted $20^{\circ}$ towards the detector, giving an X-ray take-off angle of $34^{\circ}$. Standards used to obtain the $\mathrm{K}$-factors for the transformation of intensity ratios to concentration, following the methods of Cliff \& Lorimer (1975) and Champness et al. (1981), were: albite, biotite, spessartine, muscovite, olivine, titanite, $\mathrm{CaSO}_{4}$ and $\mathrm{MnSO}_{4}$. The ultra-thin window detector allows oxygen qualitative identification, but since its number of X-ray counts is strongly affected by small differences in sample thickness, it was not quantitatively measured. Atomic concentration ratios were converted into formulae according to stoichiometry (number of oxygens in the theoretical formulae of minerals). Alkali loss is a significant problem in the TEM analysis of clay minerals, particularly for defect-rich minerals (Van der Pluijm et al., 1988). The comparison of analyses obtained for 15 to $100 \mathrm{~s}$ showed that shorter counting times gave improved reproducibility for these elements; therefore, counting times of $15 \mathrm{~s}$ were used as a compromise for major alkali analysis (Nieto et al., 1996).

\section{TEM RESULTS}

A synthesis of the results obtained in the TEM study is presented in Table 2, while the AEM results are detailed in Tables 3, 4, 5 and 6, for mica, chlorite, smectite and mixed-layer clay minerals, respectively.

\section{Lattice-fringe images and electron diffraction patterns}

The phyllosilicates identified in all samples were dioctahedral mica and chlorite + smectite in several cases. More common defects are low-angle boundaries between packets of the same mineral (Fig. 3) or between mica and chlorite packets; less frequently, coherent adjacent packets can also be observed. The dominant polytype in dioctahedral micas is $2 M$, although $1 M d$ is also present in appreciable amounts (Table 2); in a few cases, partial $10 \AA$ periodicity occurs in $\mathrm{k} \neq 3 n$ rows within a line of streaking (Fig. 3, inset). Muscovite occurs as defect-free packets with straight and in general continuous 10 or $20 \AA$ lattice fringes, and the typical mottled texture (Fig. 4). Besides, phengite with three-layer sequences, interpreted as $3 T$ polytype were identified in two samples (Fig. 5a). Some isolated layers at $14 \AA$ and at $24 \mathrm{~A}$, interleaved inside dominant mica packets, were observed in a few cases. Mica packet thickness varies considerably in each sample (Table 2). In slates PU3, PU4, PU20, PU42 and PU48, thin mica packets in the range 200-500 predominate. Furthermore, the distribution is skewed towards greater thickness. No sharp break in thickness distribution that could be attributed to the occurrence of detrital mica was registered. Mica packets of $>2000 \AA$, which could correspond to detrital mica, are absent or represent $<10 \%$ of the 


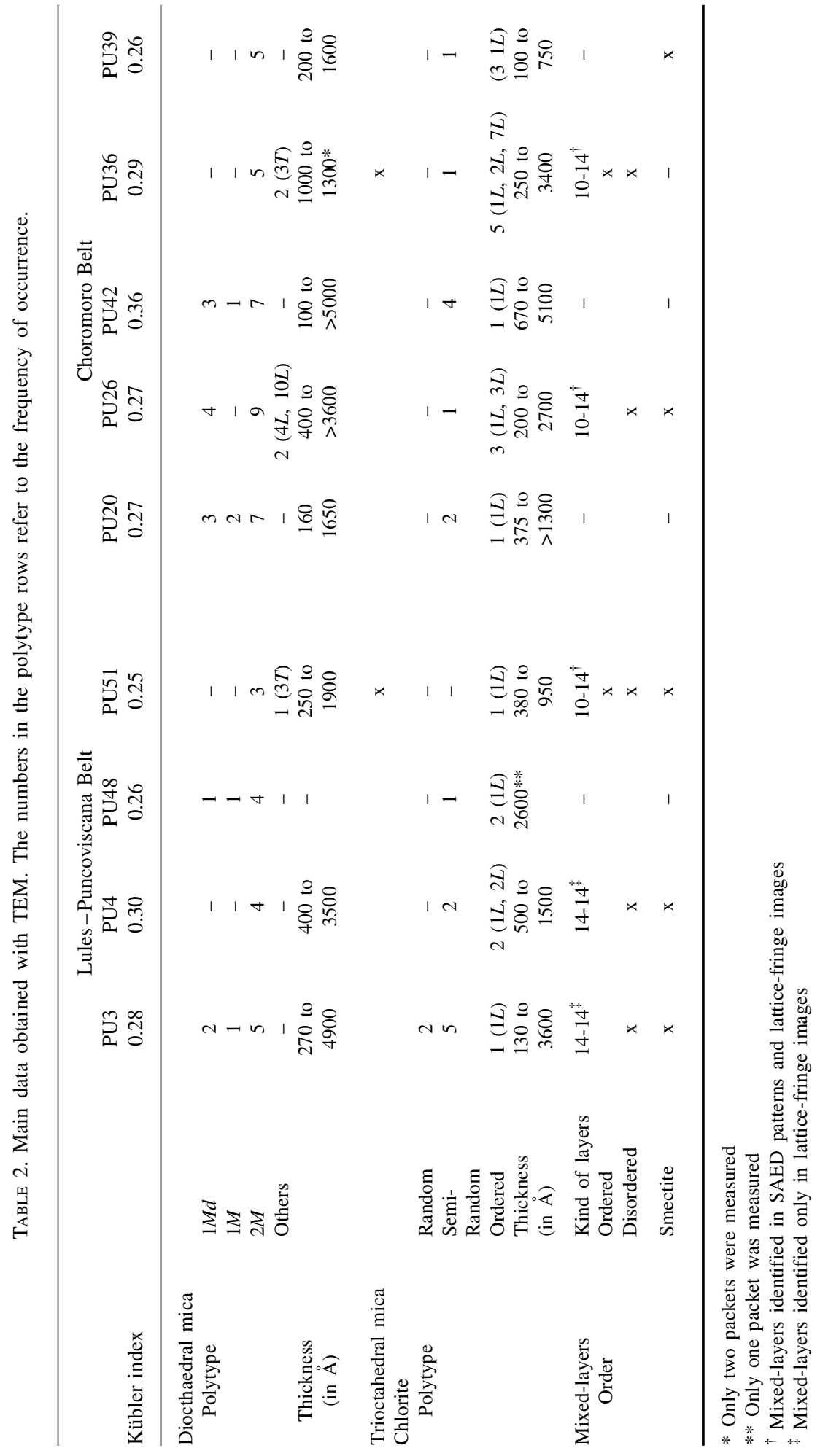




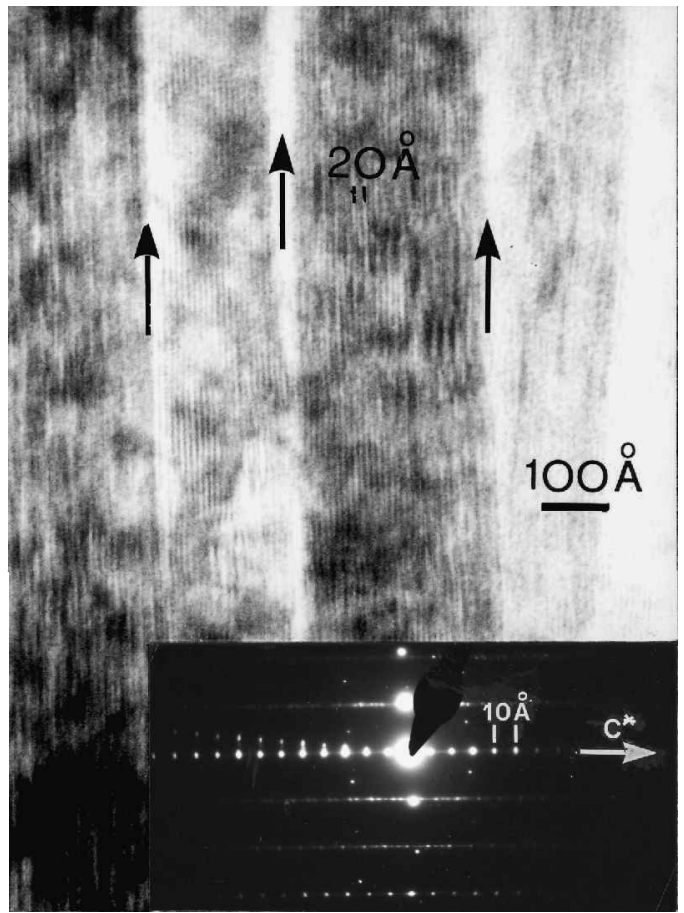

FIG. 3. SAED pattern view along [100] (inset) and lattice-fringe image of $1 M$ partially disordered phengite. Arrows indicate low-angle boundaries between packets. Sample PU3.

mica population. In samples PU26, PU36 and PU39, mica packets thousands of $\AA$ thick are more abundant.

An interlayer-deficient trioctahedral mica $1 M d$ polytype, distinguished from muscovite by AEM (PU51-6, in Table 3), was identified in sample PU51. Because its predominant interlayer cation was $\mathrm{Na}$, it could be termed wonesite. In the latticefringe image, three packets of mica 25,50 and 75 layers thick with straight and continuous $10 \AA$ lattice fringes could be recognized. AEM analysis revealed the presence of smectite associated with this wonesite crystal.

Two SAED patterns representing long-range mica stacking sequences were obtained in sample PU26. One of them had points with a $40 \AA$ periodicity in $\mathrm{k}$ $\neq 3 n$ rows of reflections and a $10 \AA$ periodicity in $\mathrm{k}=3 n$ rows, i.e. a four-layer stacking sequence (Fig. 6a); in some cases three successive points display the same intensity, while the fourth is fainter. The corresponding lattice-fringe image illustrates a packet more than $1000 \AA$ thick with straight and continuous $10 \AA$ lattice fringes.

The other long-range mica stacking sequence shows reflections corresponding to a $100 \AA$ periodicity in $\mathrm{k} \neq 3 n$ rows, whereas the $\mathrm{k}=3 n$ reflections have a $10 \AA$ periodicity, i.e. a ten-layer stacking sequence (Fig. 6b). This muscovite occurs as defect-free packets with straight $10 \AA$ lattice fringes, except for abundant dislocations that interrupt the basal planes in two packets.

Chlorite with both semi-random and ordered stacking were identified in SAED patterns; ordered polytypes prevail in samples PU39, PU36, PU26, PU48 and PU51, while in PU3, PU4, PU42 and PU20 semi-random stacking predominates. PU3 is the only metapelite in which disordered chlorite was also identified. In general, chlorite exhibits straight and continuous, defect-free $14 \AA$ lattice fringes (Fig. 7). Figure 8 reveals one packet of semi-random chlorite $>2600 \AA$ thick, no open layers were observed, and the only evidence of disorder is the existence of scarce interleaved $10 \AA$ and $24 \AA$ layers. In chlorite from samples PU3 and

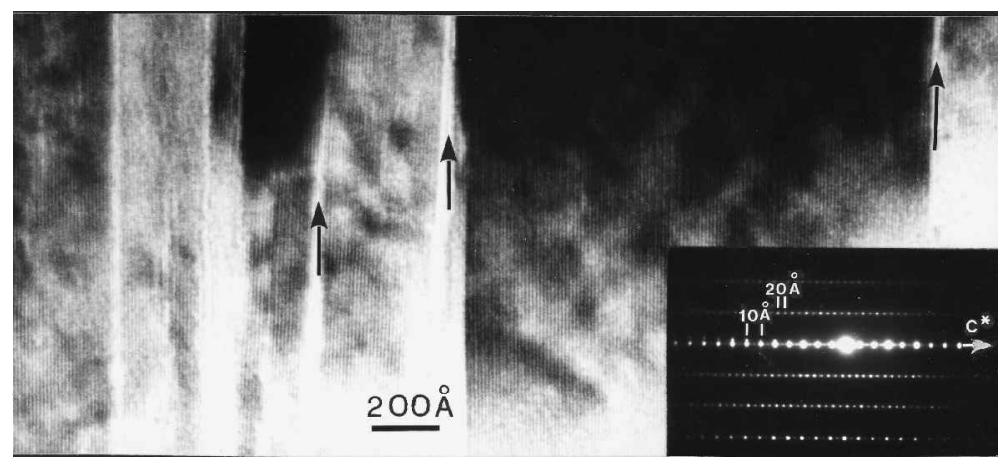

FIG. 4. SAED pattern view along 110 (inset) and lattice-fringe image of $2 M$ muscovite showing $10 \AA$ layers. Arrows indicate low-angle boundaries between packets. Sample PU26. 


\begin{tabular}{|c|c|c|c|c|c|c|c|}
\hline $\begin{array}{l}\frac{ \pm}{1} \\
\frac{n}{2}\end{array}$ & $\begin{array}{l}0+\infty \\
\text { ri } \\
\text { ri }\end{array}$ & 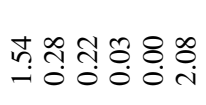 & 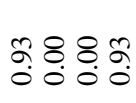 & 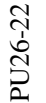 & त̂? & 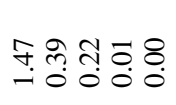 & 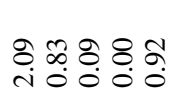 \\
\hline$\frac{0}{\frac{1}{n}}$ & $\begin{array}{l}\text { If } \\
\text { ri }\end{array}$ & †ि & $\begin{array}{l}25 \\
\stackrel{0}{0} \\
0\end{array}$ & 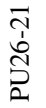 & mi? & 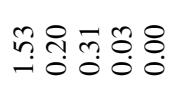 & 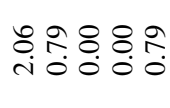 \\
\hline $\begin{array}{ll}0 & 0 \\
0 & 0 \\
0 & 1 \\
0 & 2 \\
3 & 2\end{array}$ & 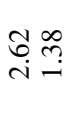 & 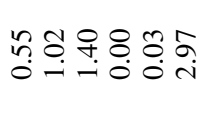 & 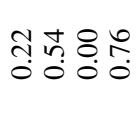 & 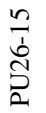 & ஸે & 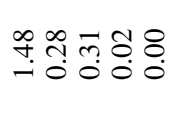 & 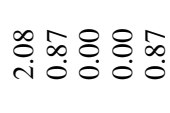 \\
\hline$\frac{1}{\frac{1}{2}}$ & ஜே & 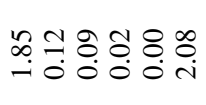 & 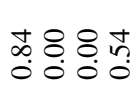 & 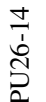 & ले & 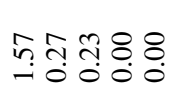 & 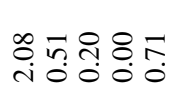 \\
\hline$\frac{7}{n}$ & 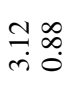 & 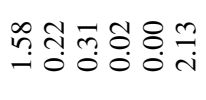 & 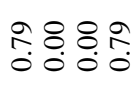 & î̀ & $\begin{array}{ll}n \\
m & 0 \\
m & 0 \\
0\end{array}$ & 市 స̂ำ & 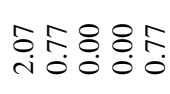 \\
\hline $\begin{array}{l}\bar{c} \\
\text { ó } \\
\dot{2} \\
0\end{array}$ & $\underset{n}{n} \overbrace{0}^{\infty}$ & 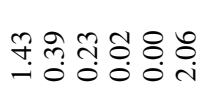 & مُ & 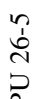 & ñ & 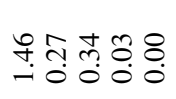 & 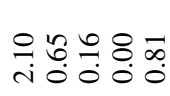 \\
\hline \begin{tabular}{l}
$\frac{n}{1}$ \\
\multirow{2}{2}{} \\
2
\end{tabular} & $\underset{g}{g} \bar{n}$ & 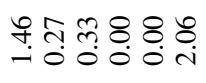 & $\begin{array}{llll}\infty & 8 & 8 & 0 \\
0 & 0 & 0 \\
0 & 0 & 0 & 0\end{array}$ & 官 & $\begin{array}{l}m \\
m \infty\end{array}$ & ป⿱艹 & 今े \\
\hline $\begin{array}{l}\frac{1}{3} \\
\frac{1}{2}\end{array}$ & $\begin{array}{l}\text { ì } \\
\text { mi }\end{array}$ & 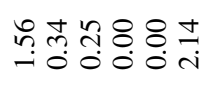 & 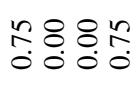 & 仓ั & $\begin{array}{l}\stackrel{\infty}{+} \stackrel{\infty}{n} \\
\text { ris }\end{array}$ & 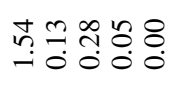 & 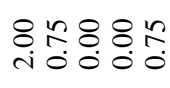 \\
\hline 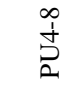 & तิ & ํ. & 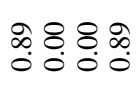 & $\begin{array}{l}\text { aे } \\
\text { ণ } \\
\text { D }\end{array}$ & \begin{tabular}{c}
\multirow{2}{*}{} \\
ri
\end{tabular} & ทิ సิ & 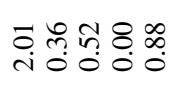 \\
\hline 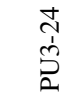 & $\begin{array}{l} \pm \infty \\
\dot{m} \infty\end{array}$ & 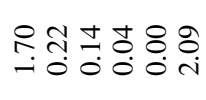 & $\begin{array}{llll}\infty & 8 & 8 & 0 \\
0 & 0 & 0 & 0 \\
0 & 0 & 0\end{array}$ & 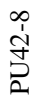 & 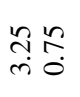 & ñ? & 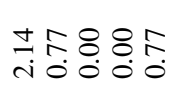 \\
\hline$\frac{n}{n}$ & 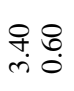 & 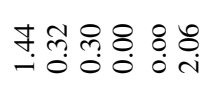 & 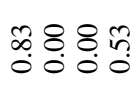 & $\begin{array}{l}\stackrel{0}{1} \\
\text { İ } \\
\text { D }\end{array}$ & $\hat{n}$ & 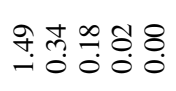 & 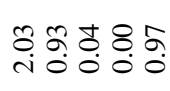 \\
\hline $\begin{array}{l}\stackrel{a}{c} \\
\grave{2}\end{array}$ & $\begin{array}{l}\hat{b} \\
\dot{n}\end{array}$ & 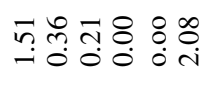 & 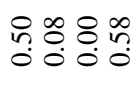 & ì & ñ & 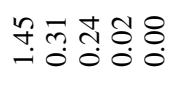 & 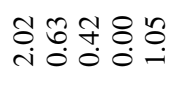 \\
\hline 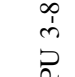 & 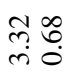 & 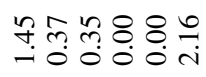 & $\begin{array}{llll}\hat{0} & 8 & 8 & 0 \\
0 & 0 & 0 \\
0 & 0\end{array}$ & 号 & 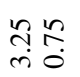 & 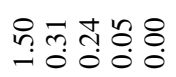 & 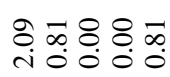 \\
\hline $\begin{array}{l}0 \\
\grave{2} \\
\grave{2}\end{array}$ & तે & లి ñ & $\begin{array}{llll}N & 8 & 0 & 0 \\
0 & 0 & 0 \\
0 & 0 & 0 & 0\end{array}$ & $\frac{n}{\frac{n}{n}}$ & $\begin{array}{l}\exists \infty \\
\text { ले }\end{array}$ & 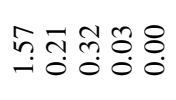 & 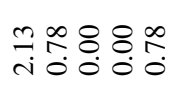 \\
\hline 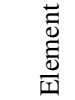 & $=\frac{2}{2}$ & 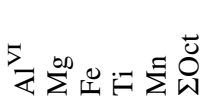 & $\triangle \tilde{Z} \tilde{U}$ & 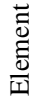 & $i \frac{2}{2}$ & 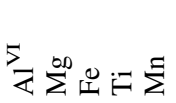 & 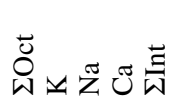 \\
\hline
\end{tabular}




\begin{tabular}{|c|c|c|c|}
\hline 官 & gे & 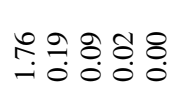 & 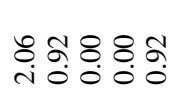 \\
\hline 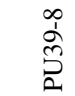 & $\begin{array}{l}0 \\
\text { mi } \\
\text { ri }\end{array}$ & 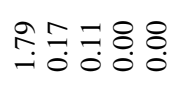 & 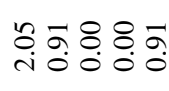 \\
\hline 傆 & $\begin{array}{l}2 \\
\dot{\infty} \\
\dot{\infty}\end{array}$ & 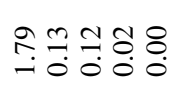 & 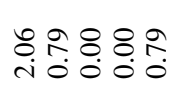 \\
\hline 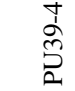 & ه: & M. & ப் \\
\hline 竞 & 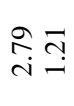 & 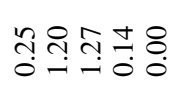 & 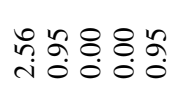 \\
\hline 仓’ & $\begin{array}{l}=\infty \\
=\infty \\
\text { ni }\end{array}$ & 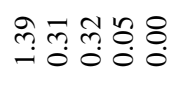 & co \\
\hline $\begin{array}{l}\bar{b} \\
\stackrel{0}{2} \\
\end{array}$ & 苾 & 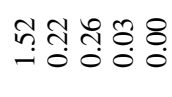 & 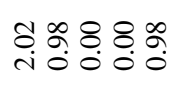 \\
\hline 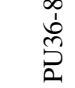 & लि? & ஜำ & 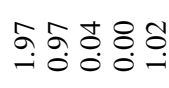 \\
\hline 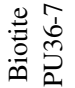 & $\begin{array}{l}\infty \\
\dot{0} \\
i\end{array}$ & 웜 & 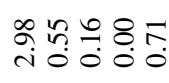 \\
\hline 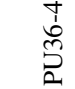 & 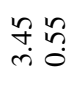 & 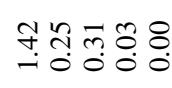 & 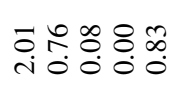 \\
\hline 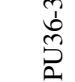 & ஸे & 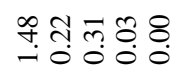 & 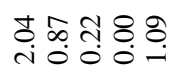 \\
\hline 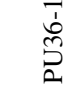 & $\underset{\sim}{\stackrel{9}{\sigma}}$ & 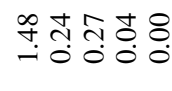 & ڤ্র் \\
\hline 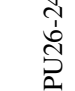 & लेश & 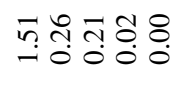 & 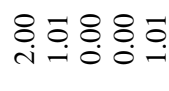 \\
\hline 峁 & 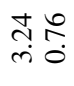 & 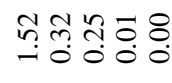 & 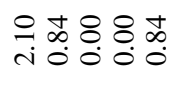 \\
\hline $\begin{array}{l}\vec{\Xi} \\
\stackrel{\overrightarrow{0}}{0} \\
\frac{\vec{D}}{\mid I}\end{array}$ & $=\frac{z}{2}$ & 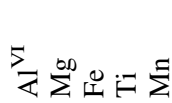 & $\stackrel{\vec{\omega}}{\dot{\omega}} \backsim \tilde{Z} \tilde{U}$ \\
\hline
\end{tabular}

PU4, dislocations changing lattice-fringe direction and occasional interleaved $10 \AA$ layers, sometimes open, were observed. Two packets of chlorite with low-angle boundaries between them are shown in Fig. 9, while in the contact, dislocations that resemble micro kink-bands appear.

Among ordered chlorites one- (Fig. 5b and Fig. 7, inset) and two-layer sequences (Fig. 5c) were identified in several samples, while a threelayer stacking sequence was only registered in slate PU26. The $3 L$ polytype chlorite presents $\mathrm{k} \neq 3 n$ reflections with a $42 \AA$ periodicity, whereas the $\mathrm{k}=$ $3 n$ reflections have a $14 \AA$ periodicity (Fig. 6c). Moreover, in slate PU36 a chlorite with long-range stacking was identified. It exhibits a $98 \AA$ periodicity in $\mathrm{k} \neq 3 n$ rows, indicating a sevenlayer stacking sequence (Fig. 5d). Only five points intercalated between $00 \mathrm{l}$ reflections were observed, probably due to low intensity in some super-order reflections. Ordered as well as semi-random chlorite shows straight and continuous $14 \AA$ and occasionally $28 \AA$ lattice fringes, Fig. 7 shows a defect-free packet of more than $2300 \AA$. In several packets, scarce interleaved $10 \AA$ or $24 \AA$ layers were identified, in other cases small dislocations were observed. In slate PU36 lateral changes were observed from a $14 \AA$ layer to a $10 \AA$ layer or from a $14 \AA$ layer to a $24 \AA$ layer, the latter frequently associated with dislocations.

Samples from Sierra de Guachipas and slate PU20 gave SAED patterns showing points with superimposed $10 \AA$ and $14 \AA$ periodicities (Fig. 10, inset). The corresponding HRTEM image (Fig. 10) shows thin packets of muscovite intercalated among chlorite packets, and low-angle boundaries between them. A lattice-fringe image reveals some interleaved $14 \AA$ layers within one of the muscovite packets. In western samples (PU26, PU36 and PU39) thick defect-free mica and chlorite packets are more frequent.

Biotite, distinguished from muscovite by AEM (PU36-17 in Table 3), occurs as a $1 M$ ordered polytype in sample PU36 (Fig. 11); its b parameter of $9.2 \AA$ is also consistent with a trioctahedral phyllosilicate. Figure 11 shows several packets 150 to 290 layers thick that are coherently related or exhibit low-angle boundaries with straight and continuous $10 \AA$ lattice fringes. Lateral transitions from a $10 \AA$ layer to a $14 \AA$ layer linked with dislocations could be observed.

Several packets of $10-14 \AA$ mixed layers were identified in slate PU36. Figure 5e shows a 
TABLE 4. Representative AEM analyses of chlorites.

\begin{tabular}{lcccccccccc}
\hline Element & PU3-28 & PU4-6 & PU4-7 & PU48-2 & PU48-3 & PU48-4 & PU48-5 & PU48-6 & PU20-3 & PU20-14 \\
\hline $\mathrm{Si}$ & 2.77 & 2.70 & 2.57 & 2.69 & 2.82 & 2.88 & 2.79 & 2.69 & 2.59 & 2.64 \\
$\mathrm{Al}^{\mathrm{IV}}$ & 1.23 & 1.30 & 1.43 & 1.31 & 1.18 & 1.12 & 1.21 & 1.31 & 1.41 & 1.36 \\
$\mathrm{Al}^{\mathrm{VI}}$ & 1.14 & 1.19 & 1.17 & 1.29 & 1.10 & 1.11 & 1.07 & 1.21 & 1.51 & 1.33 \\
$\mathrm{Mg}$ & 3.03 & 2.42 & 2.29 & 2.21 & 2.53 & 2.57 & 2.63 & 2.00 & 1.61 & 2.01 \\
$\mathrm{Fe}$ & 1.83 & 2.37 & 2.53 & 2.52 & 2.37 & 2.33 & 2.35 & 2.85 & 2.84 & 2.67 \\
$\mathrm{Ti}$ & 0.00 & 0.00 & 0.00 & 0.00 & 0.04 & 0.00 & 0.02 & 0.00 & 0.00 & 0.00 \\
$\mathrm{Mn}$ & 0.02 & 0.04 & 0.07 & 0.00 & 0.00 & 0.00 & 0.00 & 0.00 & 0.00 & 0.00 \\
$\Sigma \mathrm{Oct}$ & 6.02 & 6.02 & 6.06 & 6.01 & 6.04 & 6.01 & 6.07 & 6.05 & 5.95 & 6.01 \\
$\mathrm{~K}$ & 0.00 & 0.00 & 0.00 & 0.00 & 0.00 & 0.00 & 0.00 & 0.00 & 0.00 & 0.00 \\
$\mathrm{Na}$ & 0.00 & 0.00 & 0.00 & 0.00 & 0.00 & 0.00 & 0.00 & 0.00 & 0.00 & 0.00 \\
$\mathrm{Ca}$ & 0.00 & 0.00 & 0.00 & 0.00 & 0.00 & 0.00 & 0.00 & 0.00 & 0.00 & 0.00 \\
& & & & & & & & & &
\end{tabular}

Element PU26-12 PU26-26 PU36-9 PU36-6 PU36-10 PU36-18 PU36-19 PU39-7 PU39-10 PU39-14

\begin{tabular}{lllllllllll}
\hline $\mathrm{Si}$ & 2.78 & 2.65 & 2.74 & 2.70 & 2.68 & 2.74 & 2.56 & 2.68 & 2.66 & 2.64 \\
$\mathrm{Al}^{\mathrm{IV}}$ & 1.22 & 1.35 & 1.26 & 1.30 & 1.32 & 1.26 & 1.44 & 1.32 & 1.34 & 1.36 \\
$\mathrm{Al}$ & 1.30 & 1.18 & 0.97 & 1.12 & 1.19 & 1.26 & 1.22 & 1.41 & 1.47 & 1.44 \\
$\mathrm{Mg}$ & 2.10 & 2.47 & 3.02 & 2.70 & 2.86 & 2.40 & 2.43 & 1.79 & 1.94 & 1.72 \\
$\mathrm{Fe}$ & 2.56 & 2.43 & 2.13 & 2.23 & 2.22 & 2.30 & 2.40 & 2.74 & 2.50 & 2.75 \\
$\mathrm{Ti}$ & 0.00 & 0.00 & 0.00 & 0.00 & 0.00 & 0.00 & 0.00 & 0.00 & 0.00 & 0.00 \\
$\mathrm{Mn}$ & 0.00 & 0.00 & 0.03 & 0.04 & 0.00 & 0.02 & 0.03 & 0.00 & 0.02 & 0.01 \\
$\Sigma \mathrm{Oct}$ & 5.96 & 6.08 & 6.15 & 6.09 & 6.07 & 5.98 & 6.08 & 5.94 & 5.92 & 5.93 \\
$\mathrm{~K}$ & 0.00 & 0.02 & 0.00 & 0.00 & 0.00 & 0.00 & 0.00 & 0.03 & 0.00 & 0.02 \\
$\mathrm{Na}$ & 0.00 & 0.00 & 0.00 & 0.00 & 0.00 & 0.00 & 0.00 & 0.00 & 0.00 & 0.00 \\
$\mathrm{Ca}$ & 0.00 & 0.00 & 0.00 & 0.00 & 0.00 & 0.00 & 0.00 & 0.00 & 0.00 & 0.00 \\
& & & & & & & & & &
\end{tabular}

representative SAED pattern in which the following of $0.60 \%$ (Bailey, 1982) was obtained for this reflections were measured, expressed in $\AA$ : 24,12 , pattern, indicating an ordered $10-14 \AA$ mixed $7.9,7.3,6.0,4.8,4.0,3.4,3.0,2.8,2.7,2.4,2.2,2.0$, layer. Reflections at 7.3 and $2.8 \AA$ were not $1.9,1.7,1.6,1.5$ and 1.4. A coefficient of variation considered in the calculation as they probably

TABLE 5. Representative AEM analyses for smectites.

\begin{tabular}{lcccccccccc}
\hline Element & PU3-12 & PU3-14 & PU3-22 & PU4-9 & PU4-16 & PU51-7 & PU51-8 & PU26-13 & PU39-9 & PU39-16 \\
\hline $\mathrm{Si}$ & 3.79 & 4.03 & 3.60 & 3.68 & 3.63 & 3.61 & 3.67 & 3.56 & 3.41 & 3.69 \\
$\mathrm{Al}^{\mathrm{IV}}$ & 0.21 & -0.03 & 0.40 & 0.32 & 0.37 & 0.39 & 0.33 & 0.44 & 0.59 & 0.31 \\
$\mathrm{Al}$ & 1.54 & 1.40 & 1.55 & 1.04 & 0.79 & 1.21 & 1.72 & 1.59 & 1.55 & 1.62 \\
$\mathrm{Mg}$ & 0.34 & 0.43 & 0.38 & 0.39 & 0.22 & 0.41 & 0.18 & 0.29 & 0.24 & 0.13 \\
$\mathrm{Fe}$ & 0.20 & 0.18 & 0.25 & 0.72 & 0.92 & 0.50 & 0.21 & 0.25 & 0.37 & 0.19 \\
$\mathrm{Ti}$ & 0.00 & 0.00 & 0.00 & 0.00 & 0.04 & 0.01 & 0.02 & 0.00 & 0.00 & 0.02 \\
$\mathrm{Mn}$ & 0.00 & 0.00 & 0.00 & 0.00 & 0.00 & 0.00 & 0.00 & 0.00 & 0.00 & 0.00 \\
$\Sigma$ Oct & 2.08 & 2.01 & 2.18 & 2.15 & 1.96 & 2.14 & 2.12 & 2.13 & 2.16 & 1.96 \\
$\mathrm{~K}$ & 0.30 & 0.30 & 0.49 & 0.05 & 0.31 & 0.10 & 0.12 & 0.09 & 0.23 & 0.12 \\
$\mathrm{Na}$ & 0.00 & 0.00 & 0.00 & 0.12 & 0.18 & 0.24 & 0.00 & 0.26 & 0.13 & 0.20 \\
$\mathrm{Ca}$ & 0.00 & 0.04 & 0.00 & 0.04 & 0.09 & 0.01 & 0.00 & 0.00 & 0.00 & 0.10 \\
$\Sigma \mathrm{Int}$ & 0.30 & 0.34 & 0.49 & 0.21 & 0.58 & 0.36 & 0.12 & 0.35 & 0.36 & 0.42 \\
& & & & & & & & & & \\
\hline
\end{tabular}


TABLE 6. AEM analyses of mixed-layer minerals, structural formulae calculated for $25(14+11)$ oxygens per formula unit.

\begin{tabular}{lcccc}
\hline Element & PU3-26 & PU26-11 & PU51-2 & PU36-20 \\
\hline $\mathrm{Si}$ & 6.12 & 6.11 & 5.84 & 5.89 \\
$\mathrm{Al}^{\mathrm{IV}}$ & 1.88 & 1.89 & 2.16 & 2.11 \\
$\mathrm{Al}^{\mathrm{VI}}$ & 1.26 & 1.68 & 1.59 & 1.63 \\
$\mathrm{Mg}$ & 4.22 & 3.64 & 3.23 & 4.43 \\
$\mathrm{Fe}$ & 3.68 & 3.87 & 3.81 & 2.87 \\
$\mathrm{Ti}$ & 0.00 & 0.00 & 0.02 & 0.05 \\
$\mathrm{Mn}$ & 0.06 & 0.00 & 0.00 & 0.00 \\
$\Sigma \mathrm{Oct}$ & 9.21 & 8.99 & 8.85 & 8.97 \\
$\mathrm{~K}$ & 0.09 & 0.14 & 0.59 & 0.45 \\
$\mathrm{Na}$ & 0.00 & 0.00 & 0.65 & 0.00 \\
$\mathrm{Ca}$ & 0.00 & 0.04 & 0.00 & 0.00 \\
$\Sigma \mathrm{Int}$ & 0.09 & 0.18 & 1.24 & 0.45 \\
$\mathrm{Fe}+\mathrm{Mg} / \mathrm{Al}$ & 2.52 & 2.05 & 1.88 & 1.95 \\
$\mathrm{Si} / \mathrm{Al}$ & 1.95 & 1.71 & 1.56 & 1.58 \\
& & & & \\
\hline
\end{tabular}

correspond to small chlorite packets interleaved with the mixed-layer and recognized in the corresponding lattice-fringe image. The SAED pattern, whose orientation is $\mathbf{c}^{*}-\mathbf{b} *$, presents reflections with a $24 \AA$ periodicity in all levels, suggesting ordered stacking and a coherent crystallographic relation between the two types of layers. The value of the b parameter $(9.2 \AA)$ indicates a trioctahedral phyllosilicate. The HRTEM image displays two packets, 600 and $900 \AA$ thick, with straight and continuous apparent $24 \AA$ layers. An intermediate lattice fringe corresponding to the 10 and $14 \AA$ layers can sometimes be recognized. The $10-14 \AA$ unit is repeated up to seven successive times in one packet and ten times in the other. Interleaved between these thin, mixed-layer 'packets', one or two $14 \AA$ layers appear. Microanalysis of these packets (PU36-20 in Table 6) displays $\mathrm{Fe}+\mathrm{Mg} / \mathrm{Al}$ and $\mathrm{Si} / \mathrm{Al}$ ratios close to the values for a theoretical biotite 0.5/ chlorite 0.5 mixture. Therefore, the chemical composition as well as the $\mathbf{b}$ parameter suggest a trioctahedral character not only for the $14 \AA$ layers, but also for the $10 \AA$ layers. The low interlayer charge in the structural formulae could be explained by the excess chlorite layers observed in the latticefringe image in addition to some loss of $\mathrm{K}$.

The SAED patterns indicative of $10-14 \AA$ mixed layers were also identified in slate PU51 (PU51-2 in Table 6), the AEM analyses indicate a mixed-layer mineral formed of chlorite-like layers and trioctahedral $10 \AA$ layers. Minor amounts of random $10 \AA$ / $14 \AA$ mixed layers were also identified in slate PU26 (PU26-11 in Table 6). In this case AEM analysis displays an $\mathrm{Fe}+\mathrm{Mg} / \mathrm{Al}$ ratio slightly higher than chlorite and a high $\mathrm{Si} / \mathrm{Al}$ ratio. These facts, in addition to the low alkali content, are compatible with a chlorite-smectite mixed layer. These mixed-layer minerals will be described in detail in a separate paper (Do Campo \& Nieto, in prep.).

Smectite that occurs in several slates is always in packets clearly discordant with other phyllosilicates or in pores of the rock. Discrete smectite packets, subparallel to other phyllosilicates, were not observed. The dominant interlayer cation could be $\mathrm{K}$ or $\mathrm{Na}$; Ca present in minor amounts or is absent.

In spite of its higher IC, at TEM scale sample PU42 is similar to sample PU20, although they differ in their packet-thickness distribution. Mica packets of 250-500 $\AA$ are predominant in sample PU20, while packets up to $250 \AA$ prevail over those in the range 250-500 $\AA$ in PU42. In addition, in slate PU42 there are some subordinate mica packets thicker than $1100 \AA$.

\section{AEM composition of phyllosilicates}

Dioctahedral micas. Table 3 shows representative analyses of dioctahedral micas. In the interlayer charge-Si/Al diagram (Fig. 12a), illitic substitution can be recognized by the relationship between the low interlayer charge and the replacement of Si by Al. Illitic substitution is clearly present in some micas of samples PU3 and PU4 and in minor amounts in slate PU26.

Several mica analyses belonging to different samples show different degrees of paragonitic substitution. Two analyses (PU42-9, PU20-9, see Table 3) stand out due to their $\mathrm{Na} /(\mathrm{K}+\mathrm{Na})$ ratios of 0.60 and 0.40 . These intermediate $\mathrm{Na}-\mathrm{K}$ micas will be discussed in detail in a later section, because the existence of a miscibility gap in the muscoviteparagonite system has been well corroborated based on natural and experimental data (Guidotti, 1984; Guidotti et al., 1994a).

Analyses in general show a large variation in the $\mathrm{Fe}+\mathrm{Mg}$ contents of dioctahedral micas within each sample. In the (Fe $+\mathrm{Mg})-\mathrm{Si}$ diagram (Fig. 12b), most of the analyses plot above the line for ideal Tschermak substitution $\left(\left(\mathrm{Mg}, \mathrm{Fe}^{2+}\right)^{\mathrm{VI}}, \mathrm{Si}^{\mathrm{IV}}=\mathrm{Al}^{\mathrm{VI}}\right.$, $\mathrm{Al}^{\mathrm{IV}}$ ), indicating that, besides phengitic substitution, 


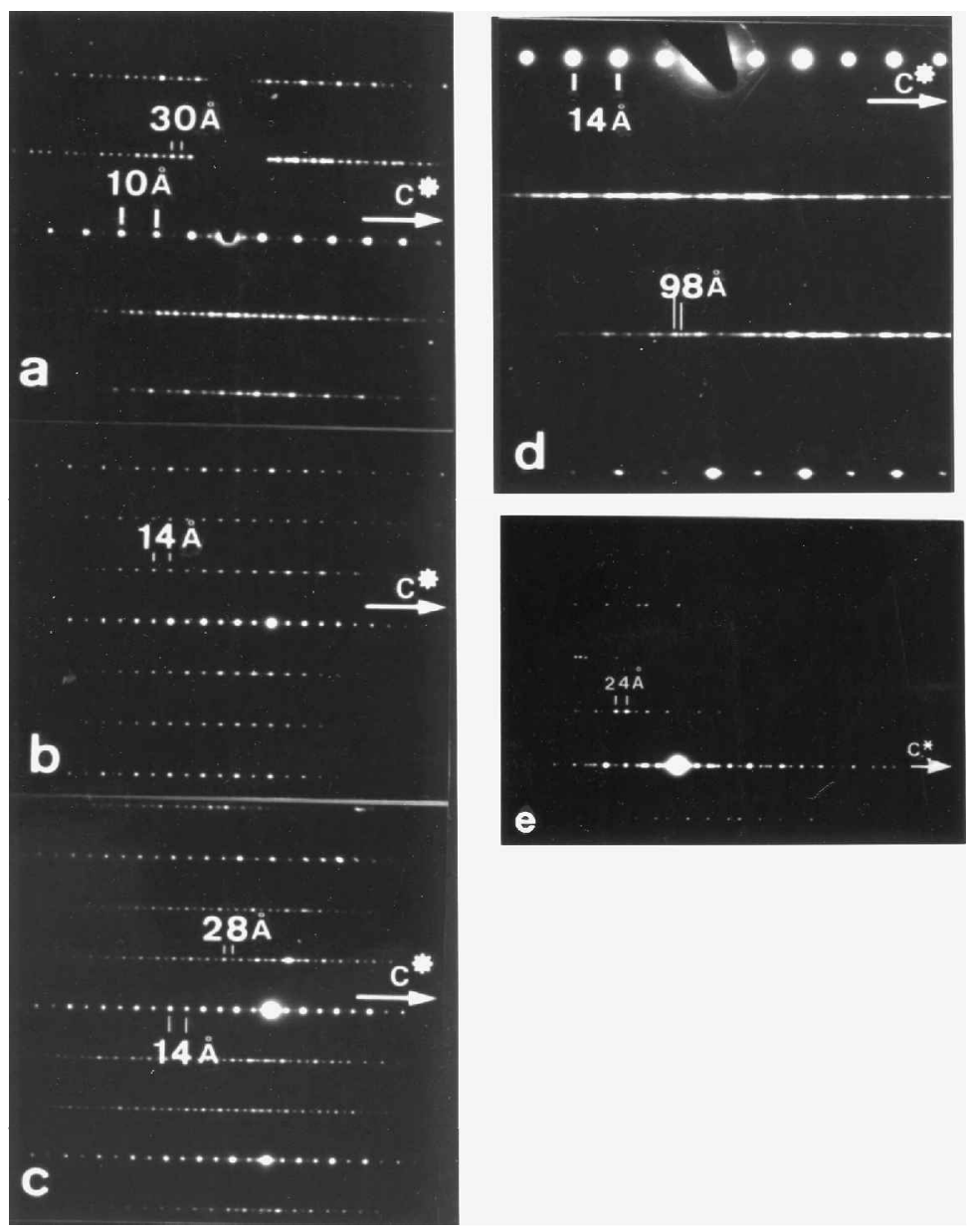

FIG. 5. SAED patterns. (a) [100] image of muscovite three-layer polytype; (b) [100] image of ordered chlorite one-layer stacking sequence; (c) [100] image of ordered chlorite two-layer stacking sequence; (d) ordered chlorite, probably seven-layer stacking sequence; and (e) [100] or equivalent image of $10 \AA-14 \AA$ mixed layer. Sample PU36.

a ferrimuscovite ( $\mathrm{Fe}^{3+}$ substituting for $\mathrm{Al}$ ) component is also present. Guidotti et al. (1994b) demonstrated that even in medium redox parageneses, containing ilmenite + magnetite, the $\mathrm{Fe}^{3+} /$ $\mathrm{Fe}_{\text {tot }}$ ratio is $>0.60$. Although magnetite was positively identified only in one case, in most of the samples ilmenite was identified by SEM, and hematite by XRD, which also indicate oxidizing conditions. Therefore, the structural formulae in Table 3 were corrected according to $\mathrm{Fe}^{+3} / \mathrm{Fe}_{\text {tot }}=$ 0.70 . This correction produced a more plausible sum of octahedral cations.

Thus, with the exception of samples PU3 and PU4, dioctahedral micas indicate a low illitic component, with phengitic and ferrimuscovite substitutions being more relevant.

The frequency distributions of the Si contents of phengites are shown in Fig. 13a,b. Only AEM analyses with an interlayer charge of $>0.75$ atoms per formula unit (a.p.f.u.) were included in the histograms to avoid drastic effects of illitic substitution, which also produce excess $\mathrm{Si}$. In addition, analyses with an octahedral sum $>2.15$ were not included. b parameter values from Do Campo (1999a,b) were included in the lower part of the figure for comparison (Fig. 13). To evaluate the effect of ferrimuscovitic substitution on white mica b-geobarometry the correlation between Si contents 


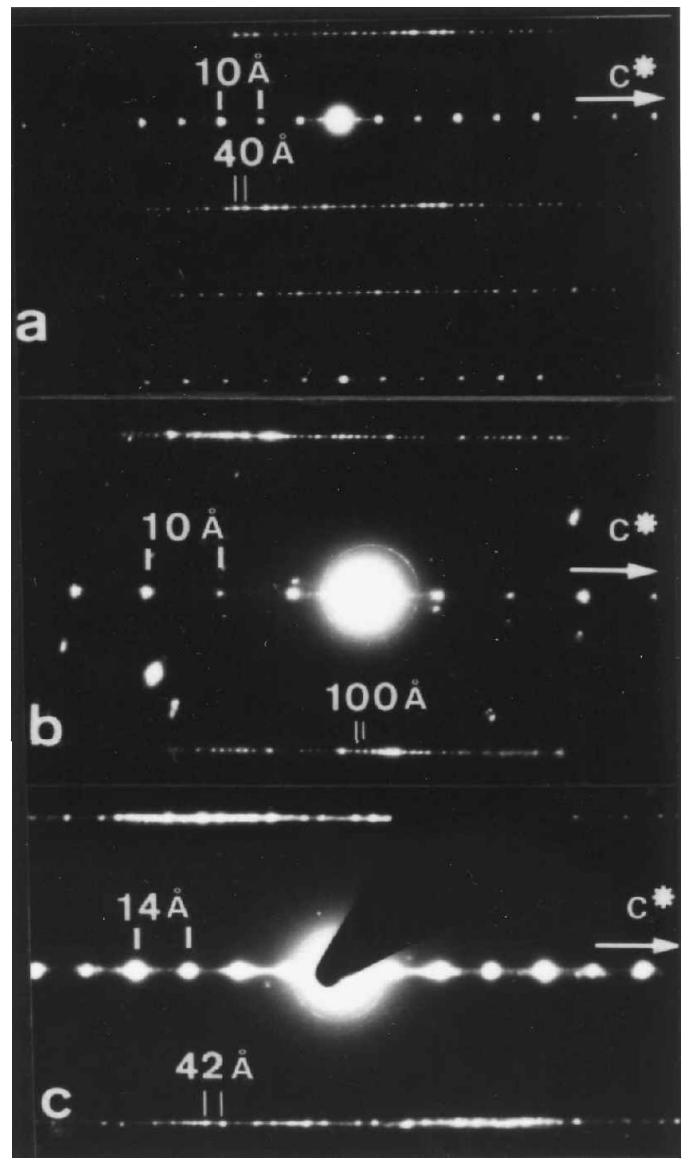

FIG. 6. SAED patterns. (a) [100] image of muscovite four-layer polytype, depicting superstructure reflections with $40 \AA$ periodicities in rows with $\mathrm{k} \neq 3 n$; (b) [100] or equivalent image of a muscovite ten-layer polytype; $100 \AA$ periodicities are visible in $\mathrm{k} \neq 3 n$ rows; (c) [100] or equivalent image of ordered chlorite three-layer polytype, $42 \AA$ periodicities are apparent in $\mathrm{k} \neq 3 n$ rows. Sample PU26.

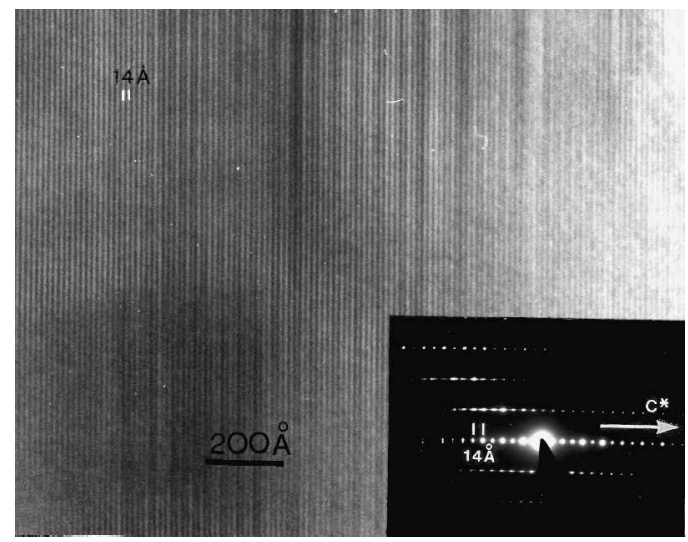

FIG. 7. SAED pattern view along [100] (inset) of chlorite 1-layer polytype and corresponding latticefringe image of a defect-free packet showing $14 \AA$ layers. Sample PU26.

and b parameter data from Do Campo (1999b) was carried out in two ways. Firstly, using the formulae of Guidotti et al. (1989), to calculate the b parameter from $\mathrm{Si}$ contents shown as open bars. Secondly, we employed the formulae of Guidotti et al. (1992), based on $\mathrm{Fe}+\mathrm{Mg}$ contents, to calculate b parameters shown as grey bars (Fig. 13). The higher $\mathbf{b}$ values obtained in the second case are a consequence of the high $\mathrm{Fe}^{3+}$ content of these phengites, i.e. the b parameter is affected not only by phengitic but also by ferrimuscovite substitution (Guidotti et al., 1989).

The Si contents of phengite from the LulesPuncoviscana belt varies between 3.06 and 3.50 exhibiting a weak maximum in the range $3.10 \leqslant \mathrm{Si}$ $<3.15$ (Fig. 13). On the other hand, the $\mathrm{Si}$ in phengite from the Choromoro belt varies from 3.00 to 3.45 , exhibiting a normal distribution, they define

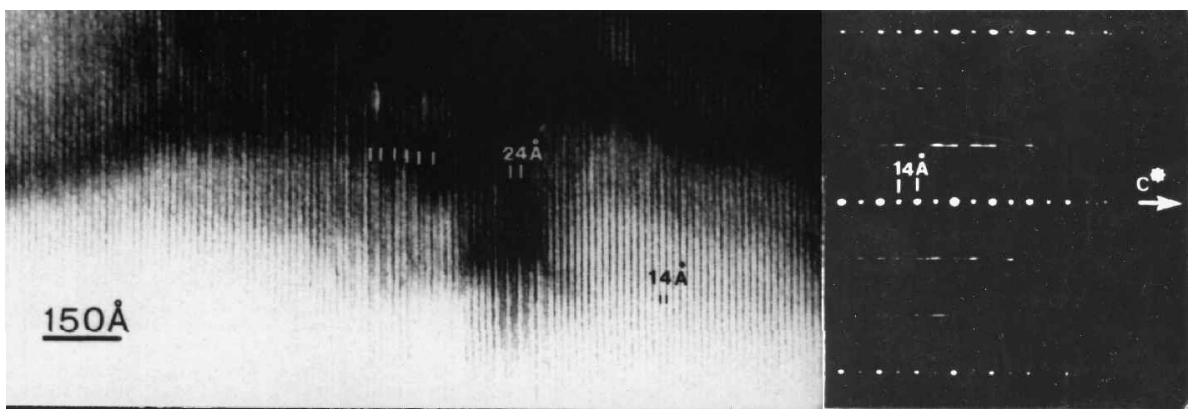

FIG. 8. SAED pattern view along [100] (inset) and lattice-fringe image of semi-random chlorite showing $14 \AA$ layers and a few interleaved $10 \AA$ (short white lines) and $24 \AA$ layers. Sample PU48. 


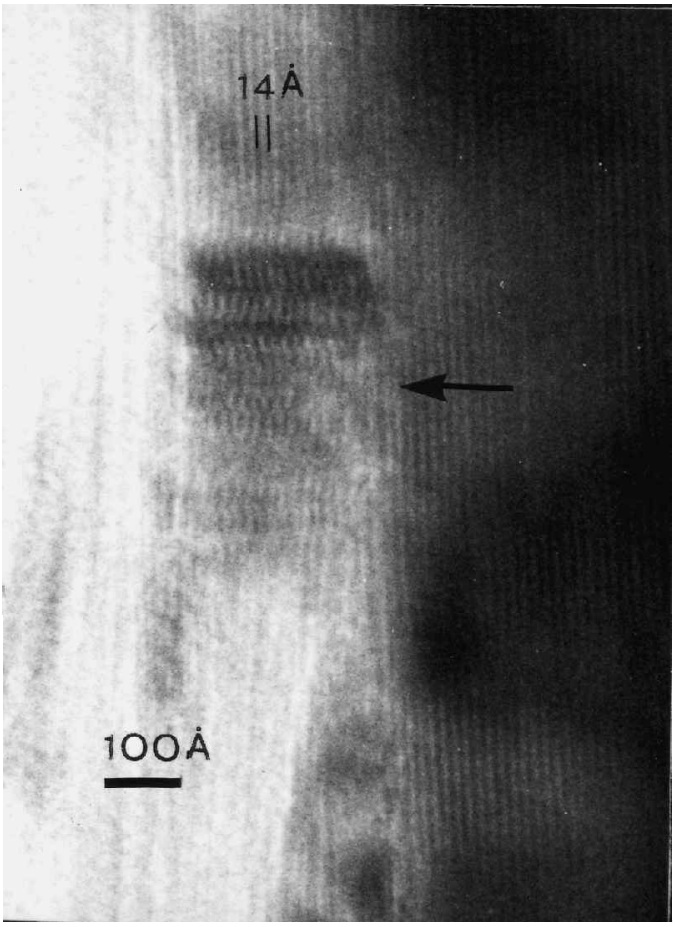

FIG. 9. Lattice-fringe image of two chlorite packets with low-angle boundaries between them showing $14 \AA$ layers. In the contact, dislocations in the form of micro kink-bands arise (indicated by arrow). Sample PU20.

a mode in the range of $3.25 \leqslant \mathrm{Si}<3.35$ (Fig. 13). The two highest $\mathrm{Si}$ contents of 3.42 and 3.45 are phengite from samples PU26 and PU36. Most of the $\mathrm{Si}$ values lower than the mode are in mica from sample PU39, which is in accordance with its low phengitic content proved by AEM analyses (Table 3 and Fig. 12b).

Chlorite. Table 4 shows representative analyses of chlorite. Analyses derived from packets that proved interlayering with other phyllosilicates in lattice-fringe images were not plotted. Many analyses show a trend to ideal Tschermak substitution by plotting close to the $\left(\mathrm{Al}^{\mathrm{VI}}+2 \mathrm{Ti}+\right.$ $\mathrm{Cr})-1$ vs. $\mathrm{Al}^{\mathrm{IV}}-1$ line (Fig. 14a). However, about one third of the analyses plot below this line suggesting that part of the $\mathrm{Fe}$ is present as $\mathrm{Fe}^{3+}$ substituting for $\mathrm{Al}$ in balancing the negative charge on the tetrahedral sheet.

The scatter of analyses on an $\mathrm{Al}^{\mathrm{IV}}-1$ vs. $\mathrm{Fe} /(\mathrm{Fe}$ $+\mathrm{Mg}$ ) (Fig. 14b) plot indicates no correlation between tetrahedral substitution and $(\mathrm{Fe}+\mathrm{Mg})$ contents. The variation in $\mathrm{Fe} /(\mathrm{Fe}+\mathrm{Mg})$ is slightly greater than the change in $\mathrm{Al}^{\mathrm{IV}}$. In several samples, $\mathrm{Fe} /(\mathrm{Fe}+\mathrm{Mg})$ varies by $<0.1$, suggesting that chemical equilibrium was reached. In metapelites PU3 and PU48, $\mathrm{Fe} /(\mathrm{Fe}+\mathrm{Mg})$ vary by $>0.1$, suggesting a lack of chemical equilibrium. For other samples, this evaluation was not possible because too few AEM analyses of pure chlorites were obtained.

\section{DISCUSSION}

\section{State of reaction progress}

The TEM results indicate a 'state of reaction progress' for the Puncoviscana slates consistent with medium-anchizone to epizone-grade metamorphism. This conclusion is based on the absence of I-S mixed layers, the prevalence of the $2 M$ polytype in dioctahedral mica, the thickness of the mica and chlorite packets, and the scarcity of crystalline defects (Merriman \& Peacor, 1999). Furthermore, the characteristics of the phyllosilicates in slates PU26, PU36 and PU39 correspond to the features enumerated by Peacor (1992) for epizonal slates, which, according to this author and Kisch (1987), do not differ from greenschistgrade rocks. For these samples, IC values lead to an underestimation of the metamorphic grade that, in the case of slate PU36, could be explained by the broadening effect of biotite on the $10 \AA$ peak. In the other samples no disturbing effect on the $10 \AA$ peak was detected, possibly reflecting the influence of strain features (Giorgetti et al., 2000) or a particular mica thickness distribution.

Direct size measurements made on lattice fringe images showed that mica packet thickness varies considerably in each sample (Table 2). In slates PU3, PU4, PU20, PU42 and PU48, thin mica packets in the range $200-500 \AA$ predominates while in samples PU26, PU36 and PU39 mica packets thousands of $\AA$ thick are more abundant. Averages, calculated for samples with at least ten values, vary between 785 and $1500 \AA$. Similar values were related by Nieto \& Sánchez Navas (1994) with high-anchizonal to epizonal metamorphism in a systematic study on mica crystallite sizes and illite crystallinity. However, other studies correlate this entire range of crystallite sizes with low-epizone conditions (see Fig. 2.1 in Merriman \& Peacor, 1999).

Schmidt et al. (1997), based on fluid inclusion data and stable isotope thermometry, correlated IC 


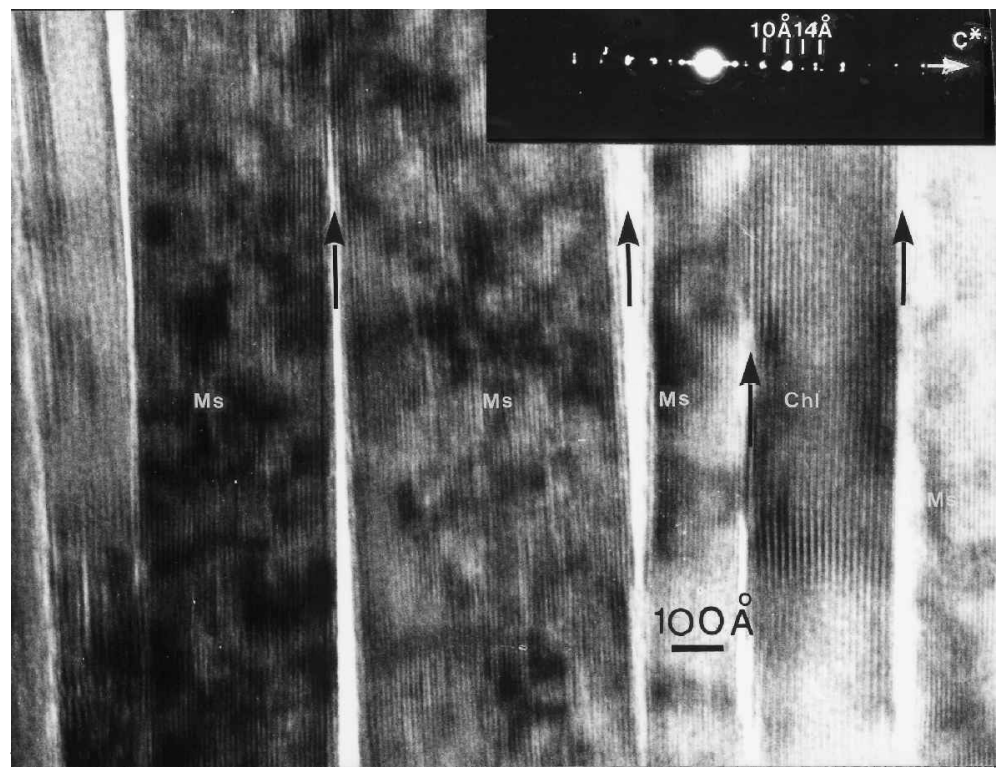

FIG. 10. SAED pattern (inset) showing reflections with superimposed $10 \AA$ and $14 \AA$ periodicities and corresponding lattice fringe showing thin packets of muscovite intercalated among chlorite packets in sample PU4. Arrows indicate low-angle boundaries.

from medium-anchizone conditions with temperatures $>270^{\circ} \mathrm{C}$. Thus, as the IC values for the Puncoviscana Formation largely correspond to high anchizone and epizone conditions, meta- morphic temperatures of $>270^{\circ} \mathrm{C}$ for peak conditions can be inferred. An independent estimate based on the concordance between K-Ar dates over fine fractions and whole rocks (Do Campo, 1999b;

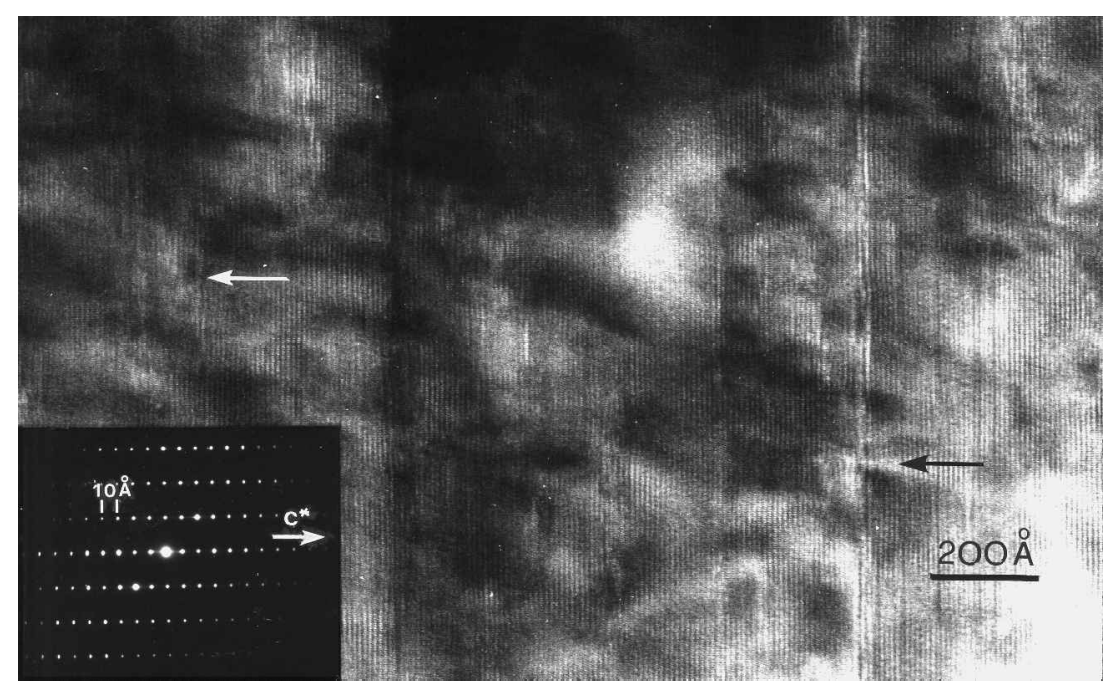

FIG. 11. SAED pattern of $1 M$ biotite view along [100] (inset) and corresponding lattice-fringe image showing $10 \AA$ layers, dislocations (black arrow) and individual layers with different orientations (indicated by white arrow). This image is bidimensional, the cross fringes correspond to $\mathbf{b}^{*}$ or 110 showing continuity across twenty layers. Sample PU36. 

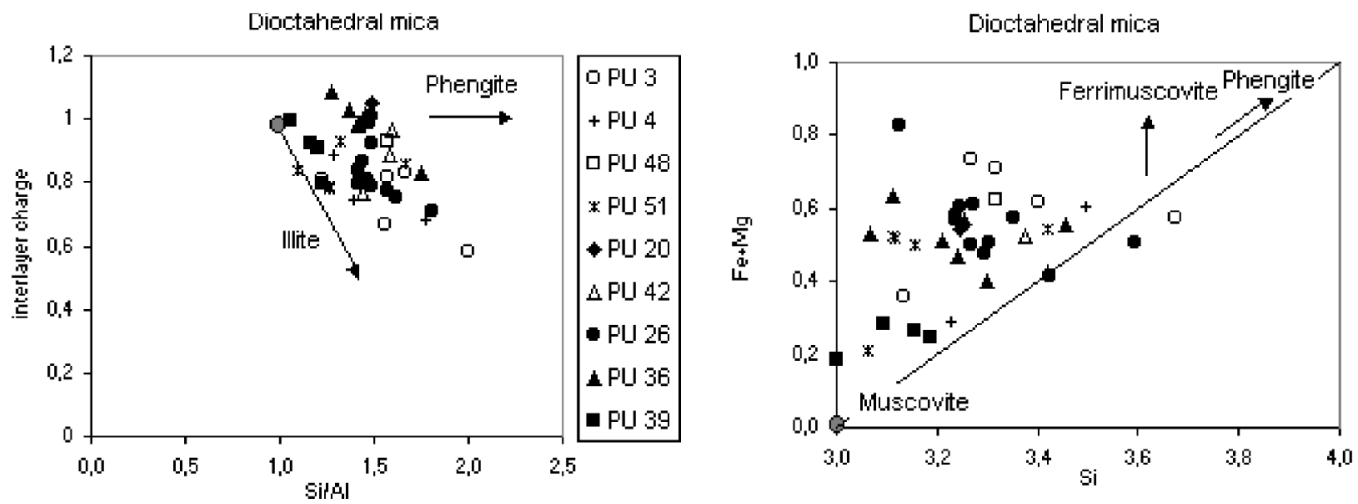

FIG. 12. Compositional diagrams for dioctahedral micas.

Do Campo et al., 1999) indicates that peak metamorphic temperatures of $\sim 350^{\circ} \mathrm{C}$ were reached at least in some localities of the basin. Moreover, the occurrence of prograde biotite in several slates from the eastern border of Puna implies temperatures of $\sim 400-450^{\circ} \mathrm{C}$ (Bucher \& Frey, 1994) for this area. Biotite formation probably occurred according to the reaction:

8 phengite + chlorite $\rightarrow$ muscovite +3 biotite +7 quartz $+\mathrm{H}_{2} \mathrm{O}$
Although the orientation of biotite flakes, parallel to slate cleavage (Fig. 2) supports this interpretation, the effect of an Ordovician thermal overprint on eastern Puna Puncoviscana rocks (Hongn et al., 2001a,b) cannot be completely ruled out.

Smectite occurring in several slates in packets clearly discordant with other phyllosilicates is interpreted as a retrograde product (Nieto et al., 1994; Zhao et al., 1999). Discrete smectite packets, subparallel to mica or chlorite, were never
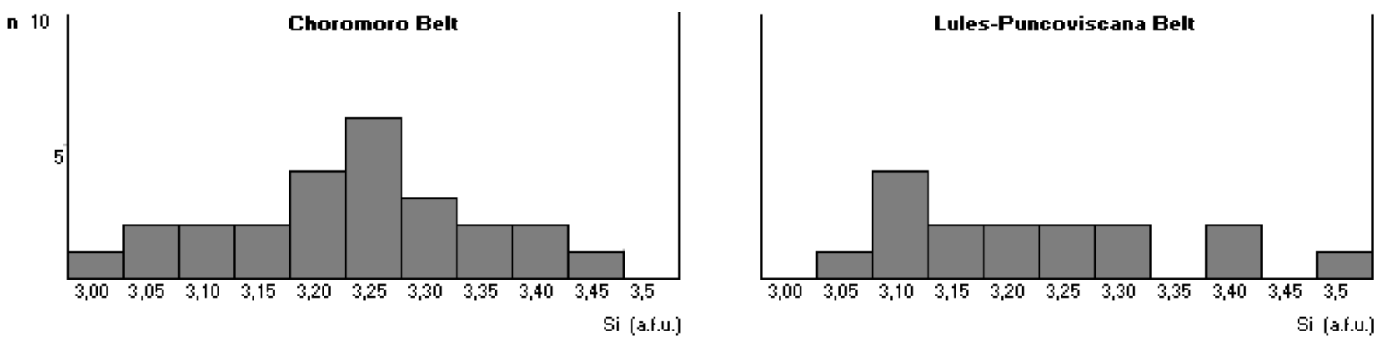

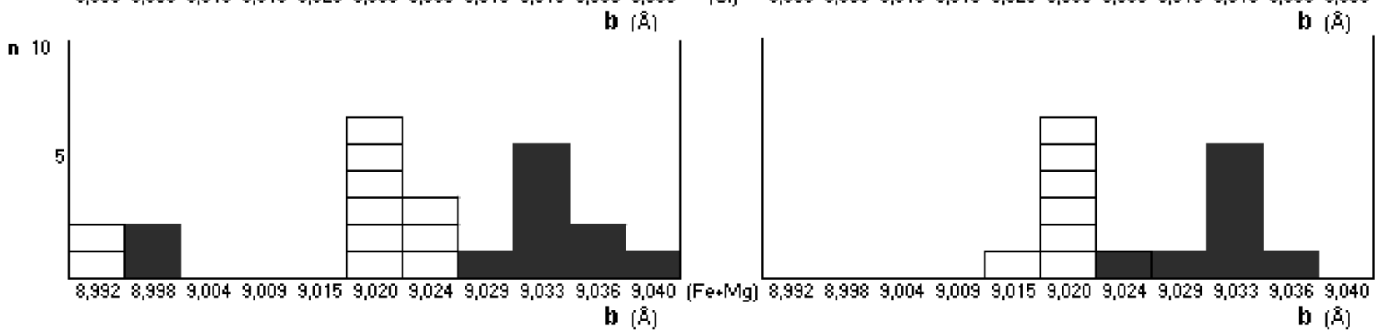

FIG. 13. Frequency distribution of Si contents of phengites from slates of Choromoro and Lules-Puncoviscana Belts, determined by AEM analyses. b parameter values from Do Campo (1999b) are shown in the lower part of the figure for comparison. Correlation between the $\mathbf{b}$ parameter and the $\mathrm{Si}$ contents were calculated from $\mathrm{Fe}+\mathrm{Mg}$ contents, grey bars (Guidotti et al., 1992) and Si contents, open bars (Guidotti et al., 1989). See text for further explanation. 
Chlorite

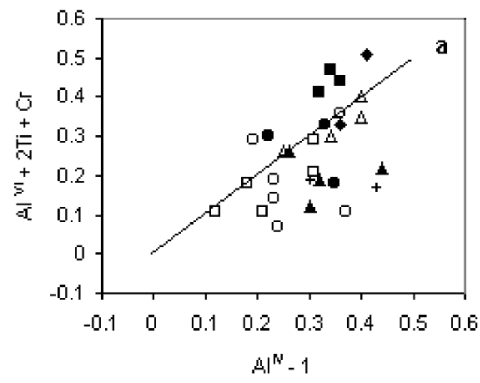

Chlorite

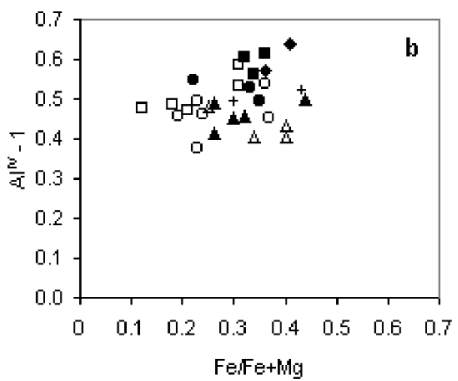

Fig. 14. Compositional diagram for chlorites (Laird, 1988). Same symbols as in Fig. 12.

observed. In addition, rare interleaved layers at $10 \AA$ were identified in some chlorite packets in samples PU3 and PU4; in a few cases layers at $10 \AA$ are more abundant, giving place to random $10 \AA / 14 \AA$ mixed layers. The occurrence of open layers in these packets, together with the $\mathrm{Si} / \mathrm{Al}$ and $(\mathrm{Fe}+\mathrm{Mg}) / \mathrm{Al}$ ratios obtained (PU3-26 in Table 6), indicated the existence of vermiculite-like layers. According to TEM and XRD observations, these slates are at least anchizonal and therefore the occurrence of individual vermiculite-like layers or chlorite/vermiculite mixed-layers is incompatible with prograde metamorphism. Their formation is more probably related to the interaction between rocks and groundwater, under oxidizing conditions, after the uplift of the Puncoviscana Formation, similar to the retrograde alteration of chlorite to smectite at a regional scale described by Nieto et al. (1994) for slates of sub-greenschist facies in Sierra Espuña (Betic Cordilleras, Spain). A 1-1 regular chlorite-vermiculite mixed layer is the predominant product of chlorite weathering in natural samples studied by Banfield \& Murakami (1998). This inference is supported by a Lower Ordovician Rb$\mathrm{Sr}$ whole-rock isochron date (Do Campo and Cagnoni 2001), discordant with previous $\mathrm{K}-\mathrm{Ar}$ dates that these authors interpreted as the time of uplift of the Puncoviscana Formation.

Crystals of mica and chlorite with features indicative of deformation, such as bending and micro kink-bands (Fig. 9), were observed at TEM scale coinciding with evidence of bending and microfolding under the optical microscope and SEM. Bearing in mind that the tectonic studies of Mon \& Hongn (1996) identified two episodes of folding in the study area, we postulate that the phyllosilicates recrystallized syntectonically during the first folding event, which produced the regional folds. Deformation features could have been produced by the second folding phase, related to the development of a crenulation cleavage, which would not produce mineral growth.

The composition of the Na-rich trioctahedral mica present in sample PU51 can be described as an intermediate member of the preiwekite-aspidolite series exhibiting a solid solution towards the $\mathrm{K}$ trioctahedral phlogopite-eastonite series. However, considering that this specimen displays a moderate interlayer deficiency we favour the name wonesite following the IMA nomenclature of micas (Rieder et al., 1998). However, the original wonesite described by Spear et al. (1981) is much more Sirich and interlayer-deficient than recorded here. Moreover, a further TEM study by Veblen $(1983 a, b)$ revealed a complex lamellar intergrowth with talc in the trioctahedral micas studied by Spear. This situation suggests that further studies will be necessary to understand the relations among Na-rich and K trioctahedral micas to establish the possible existence of miscibility gaps between them.

The chlorite-biotite mixed layers identified in slate PU36 are probably metamorphic in origin, as prograde biotite with no evidence of deformation was identified by SEM and TEM in this sample. Chlorite-biotite mixed layers probably represent a metastable product, an Ostwald intermediate step, that would probably give place to discrete biotite and chlorite in response to increasing metamorphism.

\section{Stacking sequences of phyllosilicates}

Even though the $2 M$ polytype clearly prevails in dioctahedral micas, in several cases it coexists with the $1 M d$ polytype. Optical and SEM observations 
suggest a metamorphic origin for the white mica $2 M$ polytype based on the degree of recrystallization shown by the slates. The $1 M d$ polytype usually predominates in diagenetic samples (Merriman \& Peacor, 1999). Alternatively, the very low-grade metamorphic $2 M$ polytype appears in white micas in rocks that have been subjected to stress. As the $2 M$ polytype is more abundant at higher temperatures, some authors (Velde, 1965; Sassi et al., 1994) have considered that this variable controls its stability. Nevertheless, the coexistence of these two polytypes over a wide range of temperature is probably a consequence of lack of equilibrium (López Munguira \& Nieto, 2000). Consequently, a temperature for the $1 M d$ to $2 M$ transformations could not be defined and therefore could not be employed as a reliable geothermometer.

Moreover, in the Puncoviscana Formation, the $2 M$ polytype coexists with $3 T$ phengite in two samples (PU51 and PU36) in addition, long-range four-layer and ten-layer stacking sequences were identified in another metapelite (PU26). Coexistence between $2 M$ and long-range polytypes has recently been reported by López Munguira \& Nieto (2000) for potassic white micas from epizonal samples. According to Sassi et al. (1994), the factor that stabilizes the $3 T$ polytype in metamorphic phengites is a high $P / T$ ratio.

In relation to the stacking sequences of chlorite, in samples PU39, PU36, PU26, PU48 and PU51 ordered polytypes prevail, while in PU3, PU4, PU42 and PU20 semi-random stacking predominates. PU3 is the only metapelite in which disordered chlorite was also identified. In contrast to micas, semi-random stacking is the most frequent type in chlorites. Therefore, the occurrence of many ordered chlorites $(1 L, 2 L, 3 L$ and $7 L)$ in the Puncoviscana samples is remarkable.

Factors that control the stacking sequences of phyllosilicates include pressure, temperature, the crystal growth mechanisms, mineral chemical composition and deformation history (Schmidt and Livi, 1999). Jullien et al. (1996) proposed that pressure might play an important role in the stacking sequences of cookeite from pegmatitic and metamorphic environments. These authors pointed out that ordered polytypes of cookeite behave as polymorphs remarkably sensitive to total pressure.

We should emphasize that in sample PU26, one $3 L$ chlorite and two long-range ( $4 L$ and $10 L$ ) phengites coexist. Furthermore, in sample PU36,
$2 L$ and $7 L$ chlorite as well as two $3 T$ phengites were identified. Both samples also present high $\mathbf{b}$ values (9.037 and 9.042, respectively) and high $\mathrm{Si}$ contents, as revealed by AEM, which is characteristic of intermediate-high pressure metamorphism. As suggested by Jullien et al. (1996) and Sassi et al. (1994) the total pressure of metamorphism, or the $P / T$ ratio, could in this case be the factor controlling the stacking sequences of phyllosilicates. However, the role of temperature cannot be underestimated since other investigations have proved its importance in the degree of stacking order in trioctahedral chlorites (Schmidt and Livi, 1999). The possible influence of pressure in the stacking order of chlorites is still an open question.

\section{Intermediate $\mathrm{Na} / \mathrm{K}$ dioctahedral micas}

Apparent intermediate compositions between paragonite and muscovite, similar to those reported in this work (Table 3), have been ascribed by different authors to averaging between paragonite and muscovite intergrowths at a very small scale (Shau et al., 1991), averaging of muscovite/ paragonite mixed layers (Frey, 1969), or to homogeneous metastable $\mathrm{Na} / \mathrm{K}$ muscovite (Jiang \& Peacor, 1993). More recently, Livi et al. (1997) demonstrated that XRD cannot distinguish between paragonite-muscovite mixed layers and an homogeneous, compositionally intermediate solid solution. Moreover, Livi et al. (1997) studied by TEM and EMP sample MF-925, in which Frey (1978) determined from XRD a 6:4 mixture of paragonitemuscovite along with macroscopic paragonite and muscovite. The EMP and AEM analyses of this sample indicated that mixtures of $\mathrm{Na}-\mathrm{K}$ micas exist at scales of $5 \mu \mathrm{m}$. In crystals containing sub-equal but variable amounts of $\mathrm{Na}$ and $\mathrm{K}$, X-ray maps indicated that $\mathrm{Na}$ is often concentrated on the outer edges of the grains. The Na-rich/K-rich mica boundaries in these cases were mostly normal to the apparent basal planes. With this evidence, Livi et al. (1997) postulated the existence of irregularly shaped domains of Na- and K-rich mica at scales of $<100 \AA$.

In this work, intermediate $\mathrm{Na} / \mathrm{K}$ micas were revealed by AEM analyses. These micas present straight and continuous $10 \AA$ lattice fringes and the mottled texture typical of potassic white micas. In our study we have found no evidence of different domains in intermediate $\mathrm{Na}-\mathrm{K}$ micas, which supports the hypothesis of Jiang \& Peacor (1993) 
that they constitute metastable phases that are replaced by discrete muscovite and paragonite when the metamorphic grade increases. However, we consider that our data are not conclusive. A simulation of HRTEM images of intermediate $\mathrm{Na}-\mathrm{K}$ micas at different focal conditions would be necessary to determine whether changes in contrast between Na-rich and K-rich layers allow them to be distinguished.

\section{Physicochemical evolution of phengites through a clockwise P-T-t metamorphic path}

The association of phengite in a non-limiting assemblage, with chlorite here means that the phengite barometer applying the $\mathrm{Si}$ isopleths diagram (Massone \& Schreyer, 1987) can be used to derive minimum formation pressures. For peak temperatures of $\leqslant 350-400^{\circ} \mathrm{C}$, pressures of 5 to $7 \mathrm{kbar}$ are derived for the metamorphic rocks from Choromoro belt. These values agree with facies series derived from the b values (Do Campo, 1999a), notwithstanding the fact that the contribution of ferrimuscovite substitution to this parameter has been corroborated (Fig. 13). This fact reinforces the usefulness of both methods for semi-quantitative barometric purposes. Nevertheless, numerous $\mathrm{Si}$ values lie above as well as below the cited range (Fig. 13). Phengite with the highest Si 3.42 and 3.45, from slates PU26 and PU36, which have the largest b parameters (Do Campo, 1999a), implies pressures of $<10 \mathrm{kbar}$. However, for temperatures close to $400^{\circ} \mathrm{C}$, these values point to a high-pressure metamorphism, which is unsupported by textural or mineralogical evidence. When considering these high results, it must be remembered that the accuracy of AEM analyses is less than that of the electron microprobe. Most of the Si values placed under the mode correspond to muscovites of slate PU39, in agreement with the low b parameter for this sample.

In the samples from the Choromoro belt, $56 \%$ of the phengite has $\mathrm{Si} \geqslant 3.25$. In contrast, in the Lules-Puncoviscana belt, $\mathrm{Si}$ values of $<3.25$ predominate, indicating pressures of $\sim 5 \mathrm{kbar}$. Overall, these data suggest that pressures were probably higher in the Choromoro belt than in the eastern Lules-Puncoviscana belt, which is consistent with the contrasting folding styles of these belts, mentioned above.

In several samples, dioctahedral mica with a wide range of phengitic substitution coexists, as evidenced by $\mathrm{Fe}+\mathrm{Mg}$, as well as $\mathrm{Si}$ contents. These variations could not arise from the disturbing effect of detrital white K-mica because TEM evidence indicates that they are absent or represent $<10 \%$ of the mica population. Thus, compositional variations suggest that dioctahedral micas of individual slates crystallized at different pressure conditions in response to the $P-T$ path of the metamorphism. Moreover, in several biotite-free slates (PU26, PU39, PU42), the IC values lead to an underestimation of the metamorphic grade attained in these rocks.

In a TEM-AEM and electron microprobe (EMP) study of anchizone-grade shales from the Franciscan Complex (Diablo Range, California), Dalla Torre et al. (1996) found that some white K-mica packets were zoned, having phengitic cores and muscovitic rims. These authors postulated that the metamorphic evolution of the Franciscan Complex rocks included a high-pressure/lowtemperature (HP/LT) event related to a subduction-zone tectonic regime, followed by a lowerpressure overprint possibly at higher temperatures than the HP/LT event. The HP/LT event produced small phengitic mica crystals, which, being enriched in the $<2 \mu \mathrm{m}$ fraction, would produce anchizone IC values. Therefore, the real maximum temperature responsible for the larger muscovite crystals would not be registered by the IC values. When Kisch (1987) established a general correlation between different indicators in incipient metamorphism, he emphasized that it was deduced for intermediate-pressure terrane and that therefore the behaviour of illite crystallinity in higher- or lower-pressure conditions was unknown and subject to further verification.

The coexistence of IC predominantly corresponding to anchizone and the occurrence of biotite in some slates and metavolcanic rocks intercalated in the Puncoviscana metasediments at El Niño Muerto Hill (Do Campo, 1999b) indicates a similar scenario to that described by Dalla Torre et al. (1996) for the Franciscan Complex. Phengitic mica in the Puncoviscana Formation could have formed during the maximum pressure event, which was probably followed by decompression and a rise in temperature. At that stage, muscovitic white micas and biotite would be expected to grow. Mica formed at the high-pressure stage would prevail in the $<2 \mu \mathrm{m}$ fraction, producing an IC indicative of anchizone in these rocks, as in those of the Franciscan Complex. Considering that the studied 
slates probably represent metastable equilibrium, the persistence of the former phengitic micas after the relatively $\mathrm{H} T$ event would be controlled by kinetic factors.

The association of anchizonal illite crystallinities and a clockwise $P-T$ - $t$ path, which attained highpressure conditions followed by greenschist-facies, has been recorded for the Alpujarride Complex in the Betic Cordilleras (Spain) and for the Verrucano Group in the Apennines (Italy). Although temperatures of $\sim 400^{\circ} \mathrm{C}$ and pressures of $7-9$ kbar were established for the Alpujarride Complex (Azañón \& Crespo-Blanc, 2000) these rocks had anchizone to epizone IC values (Azañón, 1994; Orozco et al., 1998).

Thermobarometric data presented by Giorgetti et al. (1998) for Verrucano metasediments in the Apennines established that they experienced relatively $\mathrm{H} P / \mathrm{L} T$ metamorphism with peak conditions ranging from $350^{\circ} \mathrm{C}$ at $8 \mathrm{kbar}$ to $420^{\circ} \mathrm{C}$ at $10 \mathrm{kbar}$. This notwithstanding, anchizone to epizone IC data were determined for these rocks (Franceschelli et al., 1991, 1994). These discrepancies between thermobarometric conditions and IC data may be a consequence of the initial $\mathrm{HP} / \mathrm{L} T$ event of the metamorphic process, as postulated in the case of the Puncoviscana Formation. The IC underestimates the metamorphic grade compared to estimates from thermobarometric data because smaller phengites formed during the $\mathrm{H} P / \mathrm{L} T$ event are concentrated in the $<2 \mu \mathrm{m}$ fraction.

Based on our data for the Puncoviscana Formation (Argentina), those from the Franciscan Complex (California) (Dalla Torre et al., 1996) and the inconsistencies in the geological literature regarding the Alpujarride Complex (Spain) and Verrucano (Italy), we conclude that high-anchizone/ low-epizone IC data of rocks in which mineral assemblages indicate higher-grade conditions may be linked with a metamorphic evolution that includes an initial relatively $\mathrm{H} P / \mathrm{L} T$ event.

The metamorphic field geothermal gradient for peak conditions of the $P-T-t$ path can be calculated from the $P-T$ conditions estimated in this study and considering the average rock density of $2960 \mathrm{~kg} / \mathrm{m}^{3}$ derived from a gravity field study of the Cordillera Oriental (Omarini et al., 1999). The gradient varies from $15^{\circ} \mathrm{C} / \mathrm{km}$ during the maximum pressure event to $26^{\circ} \mathrm{C} / \mathrm{km}$ during the decompression with a rise in temperature. The outlined metamorphic evolution of the Puncoviscana Formation would allow geodynamic models for the area to be improved.

\section{ACKNOWLEDGMENTS}

The help of M.M. Abad-Ortega (Centro de Instrumentación Científica, University of Granada) with the TEM and AEM, of J.D. Montes Rueda with the ion mill and of A. Molina Illescas with the photographic laboratory was essential for the present work. C. Laurin is responsible for the language revision of the text. Financial support was supplied by Research Project BTE 2000-0582 from the Ministerio Español de Ciencia y Tecnología and the Research Group RNM0179 of the Junta de Andalucía. The stay of M. Do Campo at the University of Granada was supported by a Unesco/ICSU/TWAS short-term fellowship awarded in 1997. The authors also thank F. Hongn and M. Pilar Mata Campo for their constructive reviews of the paper.

\section{REFERENCES}

Azañón J.M. (1994) Metamorfismo de alta presión/baja temperatura, baja presión/alta temperatura y tectónica del Complejo Alpujárride (Cordilleras Bético Rifeñas). PhD thesis, Universidad de Granada, Spain, $327 \mathrm{pp}$.

Azañón J.M. \& Crespo-Blanc A. (2000) Exhumation during a continental collision inferred from the tectonometamorphic evolution of the Alpujarride Complex in the central Betics (Alboran Domain, SE Spain). Tectonics, 19, 549-565.

Bailey S.W. (1982) Nomenclature for regular interstratifications. American Mineralogist, 67, $394-398$.

Banfield J.F. \& Murakami T. (1998) Atomic-resolution transmission electron microscope evidence for the mechanism by which chlorite weathers to $1: 1$ semiregular chlorite-vermiculite. American Mineralogist, 83, $348-357$.

Bucher K. \& Frey M. (1994) Petrogenesis of Metamorphic Rocks. Springer-Verlag, Berlin.

Cliff G. \& Lorimer G.W. (1975) The quantitative analysis of thin specimens. Journal of Microscopy, 103, 203-207.

Champness P.E., Cliff G. \& Lorimer G.W. (1981) Quantitative analytical electron microscopy. Bulletin de Minéralogie, 104, 236-240.

Dalla-Torre M., Livi K., Veblen D.R. \& Frey M. (1996) White K-mica evolution from phengite to muscovite in shales and shale matrix melange, Diablo Range, California. Contributions to Mineralogy and Petrology, 123, 390-405.

Do Campo M. (1999a) Metamorfismo del basamento en la Cordillera Oriental y borde oriental de la Puna. Pp. 41-51 in: Relatorio XIV Congreso Geológico Argentino Geología del Noroeste Argentino (G. González Bonorino, R. Omarini \& J. Viramonte, editors). Universidad de Salta, Argentina. 
Do Campo M. (1999b) Mineralogía, geoquímica y geocronolog ía de la Formación Puncoviscana

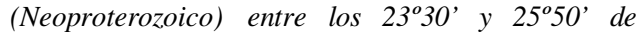
Latitud Sur, Noroeste de Argentina. PhD thesis (unpublished), Buenos Aires University, Argentina.

Do Campo M. \& Cagnoni M. (2001) The Rb-Sr isochron method applied to low-grade metamorphic rocks, the case of the Puncoviscana Formation, NW Argentina. III South American Symposium on Isotope Geology, Pucón, Actas, 26-29.

Do Campo M., Nieto F. \& Omarini R. (1998) Mineralogía de arcillas y metamorfismo de la Formación Puncoviscana en localidades de la Cordillera Oriental y Puna, Argentina. X Congreso Latinoamericano de Geología, Buenos Aires, Actas, 2, 217-223.

Do Campo M., Nieto F., Omarini R. \& Ostera H. (1999) Neoproterozoic K-Ar ages for the metamorphism of the Puncoviscana Formation, Northwestern Argentina. II South American Symposium on Isotope Geology, Carlos Paz, Actas, 48-53.

Dong H. \& Peacor D.R. (1996) TEM observations of coherent stacking relations in smectite, I/S and illite of shales: Evidence for MacEwan crystallites and dominance of $2 \mathrm{M}_{1}$ polytypism. Clays and Clay Minerals, 44, 257-275.

Franceschelli M., Leoni L. \& Sartori F. (1991) Crystallinity distribution and crystallinity- $\mathrm{b}_{0}$ relationships in white K-micas of Verrucano metasediments (Northern Apennines, Italy). Schweizerische Mineralogische und Petrographische Mitteilungen, 71, $161-167$.

Franceschelli M., Pandeli E., Puxeddu M., Porcu R. \& Fadda S. (1994) Illite crystallinity in pelitic and marly rocks from the Northern Apennines (southern Tuscany and Umbria, Italy). Neues Jahrbuch für Mineralogie Monatshefte, 8, 367-384.

Frey M. (1969) A mixed-layer paragonite/phengite of low grade metamorphic origin. Contributions to Mineralogy and Petrology, 24, 63-65.

Frey M. (1978) Progressive low-grade metamorphism of a black shale formation, Central Swiss Alps, with special reference to pyrophyllite and margarite bearing assemblages. Journal of Petrology, 19, $95-135$.

Giorgetti G., Goffé B., Memmi I. \& Nieto F. (1998) Metamorphic evolution of Verrucano metasediments in northern Apennines: new petrological constraints. European Journal of Mineralogy, 10, 1295 -1308.

Giorgetti G., Memmi I. \& Peacor D.R. (2000) Retarded illite crystallinity caused by stress-induced sub-grain boundaries in illite. Clay Minerals, 35, 693-708.

Guidotti C.V. (1984) Micas in metamorphic rocks. Pp. $357-467$ in: Micas (S.W. Bailey, editor). Reviews in Mineralogy, 13. Mineralogical Society of America, Washington, D.C.

Guidotti C.V., Mazzoli C., Sassi F.P. \& Blencoe J.G.
(1992) Compositional controls on the cell dimensions of $2 \mathrm{M}_{1}$ muscovite and paragonite. European Journal of Mineralogy, 4, 283-297.

Guidotti C.V., Sassi F.P. \& Blencoe J.G. (1989) Compositional controls on the $a$ and $b$ cell dimensions of $2 \mathrm{M}_{1}$ muscovite. European Journal of Mineralogy, 1, 71-84.

Guidotti C.V., Sassi F.P., Blencoe J.G. \& Selverstone J. (1994a) The paragonite -muscovite solvus: I. P-T-X limits derived from the $\mathrm{Na}-\mathrm{K}$ compositions of natural, quasibinary paragonite -muscovite pairs. Geochimica et Cosmochimica Acta, 58, 2269 -2275.

Guidotti C.V., Yates M.G., Dyar M.D. \& Taylor M.E. (1994b) Petrogenetic implications of the $\mathrm{Fe}^{+3}$ content of muscovite in pelitic schists. American Mineralogist, 79, 793-795.

Hongn F.D., Acuña P.A., Mon R. \& Kirschbaum A.M. (2001a) Deformación paleozoica en el área de La Colorada. Sierra de Cobres (NO de la Argentina). $X$ Congreso Argentino de Geología Económica, Salta, Actas, 1, 57-63.

Hongn F.D., Tubía J.M., Aranguren A., Mon R. \& Battaglia R. (2001b) Intrusión del granito rojo del batolito de Tastil en areniscas eopaleozoicas en el angosto de la Quesera, Cordillera Oriental, Salta. Revista de la Asociación Geológica Argentina, 56, $249-252$.

Jezek P. (1990) Análisis sedimentológico de la Formación Puncoviscana entre Tucumán y Salta. Pp. 9-36 in: El Ciclo Pampeano en el Noroeste Argentino (F.G. Aceñolaza, H. Miller \& A.J. Toselli, editors). Universidad Nacional de Tucumán, Tucumán, Argentina.

Jiang W.T. \& Peacor D.R. (1993) Fomation and modification of metaestable intermediate sodium potassium mica, paragonite and muscovite in hydrothermally altered metabasites from North Wales. American Mineralogist, 78, 782-793.

Jullien M., Baronnet A. \& Goffé B. (1996) Ordering of the stacking sequence in cookeite with increasing pressure. An HRTEM study. American Mineralogist, 81, $67-78$.

Kilmurray J.O., Merodio J.C. \& Rapela C.W. (1974) Las metamorfitas cordieríticas del área Estación Incahuasi-Santa Rosa de Tastil, Provincia de Salta: Rasgos petrológicos y geoquímicos. Revista de la Asociación Geológica Argentina, 29, 425-442.

Kisch H.J. (1987) Correlation between indicators of very low-grade metamorphism. Pp. 227-300 in: Lowtemperature Metamorphism (M. Frey, editor). Blackie, London.

Kretz R. (1983) Symbols for rock-forming minerals. American Mineralogist, 68, 277-279.

Laird J. (1988) Chlorites: metamorphic petrology. Pp. 405-496 in: Hydrous Phyllosilicates (S.W. Bailey, editor). Reviews in Mineralogy, 19. Mineralogical Society of America, Washington, D.C. 
Lee J.H. \& Peacor D.R. (1985) Ordered 1:1 interestratification of illite and chlorite: a transmission and analytical electron microscopy study. Clays and Clay Minerals, 33, $463-467$.

Lee J.H., Peacor D.R., Lewis D.D. \& Wintsch R.P. (1984) Chlorite-illite/muscovite interlayered and interstratified crystals: A TEM/STEM study. Contributions to Mineralogy and Petrology, 88, $372-385$.

Lee J.H., Peacor D.R., Lewis D.D. \& Wintsch R.P. (1986) Evidence for syntectonic crystallization for the mudstone to slate transition at Lehigh Gap, Pennsylvania, U.S.A. Journal of Structural Geology, 8, $767-780$.

Livi K.J.T., Veblen D.R., Ferry J.M. \& Frey M. (1997) Evolution of 2:1 layered silicates in low-grade metamorphosed Liassic shales of Central Switzerland. Journal of Metamorphic Geology, 15, $323-344$

López Munguira A. \& Nieto F. (2000) Transmission electron microscopy study of very low-grade metamorphic rocks in Cambrian sandstones and shales, Ossa-Morena Zone, southwest Spain. Clays and Clay Minerals, 48, 213-223.

Massone H.J. \& Schreyer W. (1987) Phengite geobarometry based on the limiting assemblage with K-feldspar, phlogopite and quartz. Contributions to Mineralogy and Petrology, 96, 212-224.

Merriman R.J. \& Peacor D.R. (1999) Very low-grade metapelites: mineralogy microfabrics and measuring reaction progress. Pp. $10-60$ in: Low-grade Metamorphism (M. Frey \& D. Robinson, editors). Blackwell Science, Oxford, UK.

Merriman R.J. Roberts B. \& Peacor, D.R. (1990) A transmission electron microscope study of white mica crystallite size distribution in a mudstone to slate transitional sequence, North Wales, UK. Contributions to Mineralogy and Petrology, 106, $27-40$.

Mon R. \& Hongn F.D. (1991) The structure of the Precambrian and Lower Paleozoic basement of the Central Andes between $22^{\circ}$ and $32^{\circ} \mathrm{S}$ Lat. Geologische Rundschau, 80, 745-758.

Mon R. \& Hongn F.D. (1996) Estructura del basamento proterozoico y paleozoico inferior del norte argentino. Revista de la Asociación Geológica Argentina, 51, 3-14.

Nieto F. \& Sánchez-Navas A. (1994) A comparative XRD and TEM study of the physical meaning of the white mica 'crystallinity' index. European Journal of Mineralogy, 6, 611-621.

Nieto F., Velilla N., Peacor D.R. \& Ortega Huertas M. (1994) Regional retrograde alteration of sub-greenschist facies chlorite to smectite. Contributions to Mineralogy and Petrology, 115, 243-252.

Nieto F., Ortega-Huertas M., Peacor D.R. \& Arostegui J. (1996) Evolution of illite/smectite from early diagenesis through incipient metamorphism in sediments of the Basque-Cantabrian Basin. Clays and Clay Minerals, 44, 304-323.

Omarini R.H. (1983) Caracterización litológica, diferenciación y génesis de la Formación Puncoviscana entre el Valle de Lerma y la Faja Eruptiva de la Puna. PhD thesis (unpublished). Salta University, Argentina, 202 pp.

Omarini R. \& Baldis B.A.J. (1984) Sedimentología y mecanismos deposicionales de la Formación Puncoviscana (Grupo Lerma, PrecámbricoCámbrico) del noroeste argentino. IX Congreso Geológico Argentino, Actas I: 384-398.

Omarini R., Sureda R.J., Götze H.J., Seilacher A. \& Pflüger F.P. (1999) Puncoviscana folded belt in the Northwestern Argentina: testimony of Late Proterozoic Rodinia fragmentation and preGondwana collisional episodes. International Journal of Earth Sciences, 88, 76-97.

Orozco M., Alonso-Chaves F.M. \& Nieto F. (1998) Development of large north-facing folds and their relation to crustal extension in the Alborán domain (Alpujarras region, Betic Cordilleras, Spain). Tectonophysics, 298, 271-295.

Peacor D.R. (1992) Diagenesis and low-grade metamorphism of shales and slates. Pp. 335-380 in: Minerals and Reactions at the Atomic Scale: Transmission Electron Microscopy (P.R. Buseck, editor). Reviews in Mineralogy, 27. Mineralogical Society of America, Washington, D.C.

Ramos V.A. \& Basei M. (1997) Gondwanan, perigondwanan, and exotic terranes of southern South America. South-American Symposium on Isotope Geology, Actas, 250-252.

Rieder M., Cavazzini G., D'yakonov Y.S., FrankKamenetskii V.A., Gottardi G., Guggenheim S., Komal P.V., Müller G., Neiva A.M.R., Radoslovich E.W., Robert J.L., Sassi F.P., Takeda H., Weiss Z. \& Wones D.R. (1998) Nomenclature of the micas. Clays and Clay Minerals, 46, 586-595.

Sassi F.P., Guidotti C.V., Rieder M. \& De Pieri R. (1994) On the occurrence of metamorphic $2 \mathrm{M}_{1}$ phengites: some thoughts on polytypism and crystallization conditions of $3 \mathrm{~T}$ phengites. European Journal of Mineralogy, 6, 151-160.

Schmidt D. \& Livi K.J.T. (1999) HRTEM and SAED investigations of polytypism, stacking disorder, crystal growth, and vacancies in chlorites from subgreenschist facies outcrops. American Mineralogist, 84, 160-170.

Schmidt D., Schmidt S.Th., Mullis J., Mahlmann R.F. \& Frey M. (1997) Very low grade metamorphism of the Taveyanne formation of western Switzerland. Contributions to Mineralogy and Petrology, 129, $385-403$.

Shau Y.H., Feather M.E., Essene E.J. \& Peacor D.R. (1991) Genesis and solvus relations of submicrosco- 
pically intergrown paragonite and phengite in a blueschist from northern California. Contributions to Mineralogy and Petrology, 106, 367-378.

Spear F.S., Hazan R.M. \& Rumble III D. (1981) Wonesite: a new rock-forming silicate from the Post Pond Volcanics, Vermont. American Mineralogist, 66, 100-105.

Toselli A.J. (1990) Metamorfismo del Ciclo Pampeano. Pp. 181 -197 in: El Ciclo Pampeano en el Noroeste Argentino (F.G. Aceñolaza, H. Miller \& A.J. Toselli, editors). Universidad Nacional de Tucumán, Tucumán, Argentina.

Toselli A.J. \& Rossi de Toselli J.N. (1982) Metamorfismo de la Formación Puncoviscana en las provincias de Salta y Tucumán, Argentina. $V$ Congreso Latinoamericano de Geología, Buenos Aires, Actas, 2, $37-52$.

Turner, J.C.M. (1960) Estratigrafía de la Sierra de Santa Victoria y adyacencias. Boletín Academia Nacional de Ciencias de Cordoba, 41, 163-196.

Van der Pluijim B.A., Lee J.H. \& Peacor D.R. (1988)
Analytical electron microscopy and the problem of potassium diffusion. Clays and Clay Minerals, 36, $498-504$.

Veblen D.R. (1983a) Exsolution and crystal chemistry of the sodium mica wonesite. American Mineralogist, 68, 554-565.

Veblen D.R. (1983b) Microstructures and mixed layering in intergrown wonesite, chlorite, talc, biotite, and kaolinite. American Mineralogist, 68, 566-580.

Velde B. (1965) Experimental determination of muscovite polymorph stabilities. American Mineralogist, 50, $436-449$.

Warr L.L. \& Nieto F. (1998) Crystalline size distributions in very low grade metamorphic pelites: a HRTEM and XRD study of clay mineral crystallinity index standards. Canadian Mineralogist, 36, $1453-1474$.

Zhao G., Peacor D.R. and McDowell D.S. (1999) Retrograde diagenesis of clay minerals in the Precambrian Freda sandstone, Wisconsin. Clays and Clay Minerals, 47, 119-130. 